\title{
Modelling and time evaluation of optical disc and retinal lesions
}

\author{
Jan Kubicek* \\ VSB-Technical University of Ostrava, \\ FEECS, K450 17. Listopadu 15, 708 33, \\ Ostrava-Poruba, Czech Republic \\ Email: jan.kubicek@vsb.cz \\ *Corresponding author \\ Juraj Timkovic \\ Clinic of Ophthalmology, \\ University Hospital Ostrava, Czech Republic \\ Email: timkovic.j@bluepoint.sk
}

\section{Marek Penhaker, Martin Augustynek, Jakub Slonka and Veronika Kovarova}

VSB-Technical University of Ostrava, FEECS, K450 17, Listopadu 15, 708 33, Ostrava-Poruba, Czech Republic

Email: marek.penhaker@vsb.cz

Email: martin.augustynek@vsb.cz

Email: jakub.slonka.st@vsb.cz

Email: veronika.kovarova.st@vsb.cz

\section{Radovan Hudak}

Faculty of Mechanical Engineering, Institute of Security and Biomedical Engineering,

Technical University of Kosice,

Kosice, Slovakia

Email: Radovan.Hudak@tuke.sk

\section{Martin Cerny}

VSB-Technical University of Ostrava, FEECS, K450 17, Listopadu 15, 708 33, Ostrava-Poruba, Czech Republic

Email: martin.cerny@vsb.cz 
Abstract: In the clinical ophthalmology, the retinal image analysis is a routine procedure with a target of the time considering the retinal lesions over the time. Unfortunately, there is an absence of the clinical software instruments providing a precise tracking the retinal lesions from the image records. Thus, the retinal image analysis is carried out by skilled physicians, but without any objective software feedback. We aim to propose a procedure having ambitions to an automatic and autonomous extraction of the retinal lesion from the retinal images, and its time evaluation. The retinal lesions are considered on a base of the optical disc which is simultaneously segmented from the retinal images. It is clinically supposed that the optical disc has stable geometrical features over the time contrarily geometrical features of the retinal lesions are timedeveloped. The proposed methodology for a time modelling of the retinal lesions comprises three essential procedures. The optical disc and the retinal lesions segmentation on a base of the time evolving curves ensure indication areas of these retinal objects. Consequently, the binary classification is used with a target of an extraction of a respective model of the optical disc and the retinal lesions.

Keywords: retinal lesions; optical disc; modelling; active snake model; binarisation; retinal image; RetCam 3; fundus camera; image processing; image segmentation; geometrical features; initial contour.

Reference to this paper should be made as follows: Kubicek, J., Timkovic, J., Penhaker, M., Augustynek, M., Slonka, J., Kovarova, V., Hudak, R. and Cerny, M. (2018) 'Modelling and time evaluation of optical disc and retinal lesions', Int. J. Digital Enterprise Technology, Vol. 1, Nos. 1/2, pp.5-36.

Biographical notes: Jan Kubicek received his MSc in Biomedical Engineering in 2012. His research interests include applied image and signal processing in medicine and statistical analysis of biomedical data.

Juraj Timkovic is the Head of the Center for Children with Visual Impairment, University Hospital Ostrava, Czech Republic and the member of the committee of the Czech Society of the Paediatric Ophthalmology and Strabismus. He holds a PhD in Paediatric Ophthalmology by the Masaryk University in Brno, Czech Republic. His research and publication interests include pediatric ophthalmology and neuro-ophthalmology.

Marek Penhaker received his MSc in Measurement and Control in $1996 . \mathrm{He}$ finished $\mathrm{PhD}$ in 2000 at VSB-TU Ostrava. His research interests include biomedical engineering, especially medical devices and home telemetry.

Martin Augustynek received his habilitation in Technical Cybernetic in 2017. His research is focused on special medical devices and diagnostic methods.

Jakub Slonka received her Bachelor degree in 2016. Her research interests include applied image processing of retinal data.

Veronika Kovarova received her Bachelor degree in 2016. Her research interests include applied image processing of retinal data.

Radovan Hudak received his MSc degree in Biomedical Engineering and his $\mathrm{PhD}$ degree in Bionics and Biomechanics from Technical University in Kosice, Slovakia. Since September 2005, he has been working as an Assistant Professor 
at Technical University in Kosice in the field of the Human Biomechanics and Medical Thermography. Throughout his career, he has published more than 120 original research articles including over 20 peer reviewed journal papers and has authored and co-authored more than eight books. His current research interests are focused on 3D printing in biomedical engineering, medical thermography, biomaterials and implantology.

Martin Cerny received his MSc in Specialisation Measurement and Control in Biomedical Engineering in 2005 and $\mathrm{PhD}$ in Technical Cybernetics with focus on biomedical engineering in 2012 at VSB-Technical University of Ostrava, Faculty of Electrical Engineering and Computer Science, Czech Republic. Nowadays, he is an Associate Professor at VSB-Technical University of Ostrava at Department of Cybernetic and Biomedical Engineering. He is specialised in biomedical engineering, telemedicine, inertial sensors and remote home care systems. He is responsible for Living Laboratory focused on modern technologies for elderly and disabled persons, which is driven by VSB-TU Ostrava.

\section{Introduction}

In the clinical ophthalmology, the retinal area is clinically evaluated on a base of either the fundus camera, or the retinal probes (RetCam 3). The main advantage of the fundus camera is the retinal image acquiring in a great resolution which typically achieves up to million pixels (Dai et al., 2017). The retinal images from the retinal fundus camera allow for a precise differentiation of the individual retinal structures with a high contrast. This fact is very important for the consequent retinal processing. In a case of the retinal probes (RetCam 3), we acquire the retinal images in a lower resolution (typically $640 \times 480$ pixels), on the other hand a great advantage of this system is the adjustable fixation to the retinal area. This tool is especially beneficial especially in cases of examination of younger patient who cannot keep themselves calm during the examination ( $\mathrm{Xu}$ et al., 2017). This device is able to capture the retinal nerve, retinal blood vessels and retinal lesions. An extraction of the optical nerve geometrical features is closely related with the retinopathy disease (Patel et al., 2017). Within this disease, it is especially important to track a time evolution of the retinal lesions in a comparison with the optical nerve which is denoted as a reference point to the respective retinal lesion. One possible clinical way for the evaluation retinal lesions is a ratio between a respective area of the retinal lesion to the optical nerve as a reference point (Fan et al., 2017).

The retinopathy of prematurity (ROP) is a vasoproliferative disease that affects especially the prematurely-born infants with a low birth weight. The ROP is the second most frequent cause of the blindness of children in developed countries (Dai et al., 2017; Xu et al., 2017). In the Czech Republic, it represents the second most frequent cause of severe children vision impairment (Oshi et al., 2017; Haq et al., 2017).

The aim of the ROP screening is an early detection of the first signs of the disease. That allows ophthalmologist to optimally schedule the ophthalmological examinations, plan a possible treatment and hereby prevent a development of the severe vision 
impairment (Patel et al., 2017). For a purpose of the clinical rating of the retinal lesions size, and for a purpose of measuring distances of the retinal changes and the assessing their progression over the time, we use the optic nerve disc diameter as a reference point. Figure 1 shows a comparison of the normal (physiological) retinal image where we can clearly recognise the retinal blood vessels and retinal structure affected by the retinopathy causing the pathological spots which have tendency to increase themselves over the time. The retinopathy goes throw by several stages in a dependence of a severity of the diseases (Munk et al., 2017). Two main pathological changes are observable. A surface of the retinal area is substantially deteriorated (Figure 2), and the pathological lesions increase themselves over the time (during individual stages) (Sharath Kumar et al., 2016).

Figure 1 Morphological comparison of (a) normal (physiologic retinal image) and (b) retinopathy image (see online version for colours)

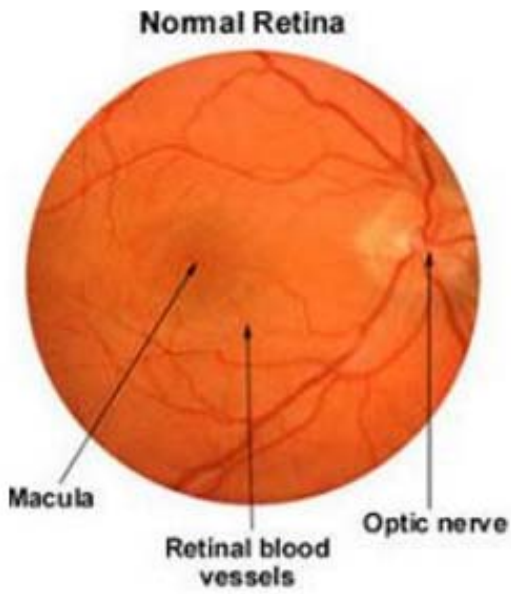

(a)

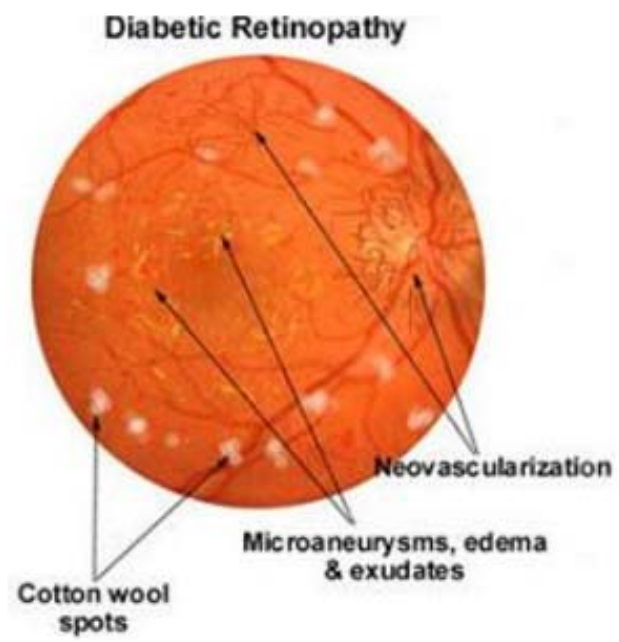

(b)

Source: Kubicek et al. (2016c)

Figure 2 Comparison of retinopathy individual changes: (a) first stage, (b) second stage and (c) third stage (see online version for colours)

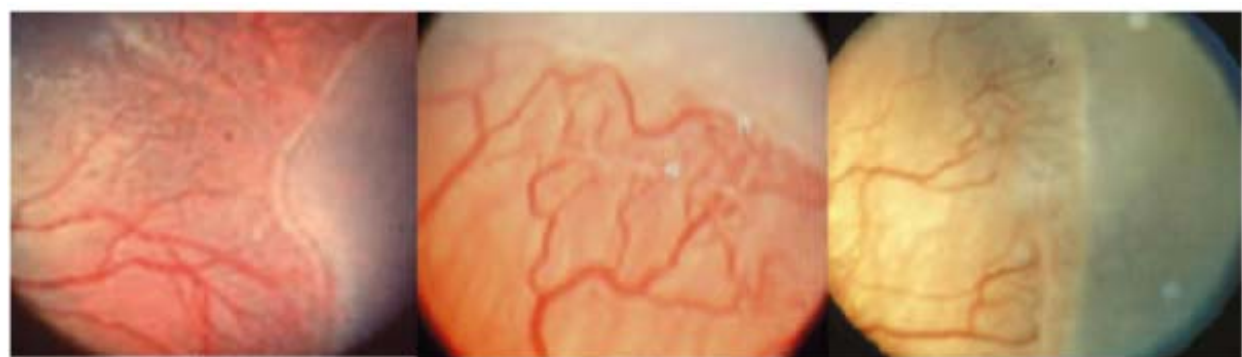

(a)

(b)

(c)

Source: Kubicek et al. (2016c) 
Figure 3 System RetCam 3 for retinal investigation (see online version for colours)

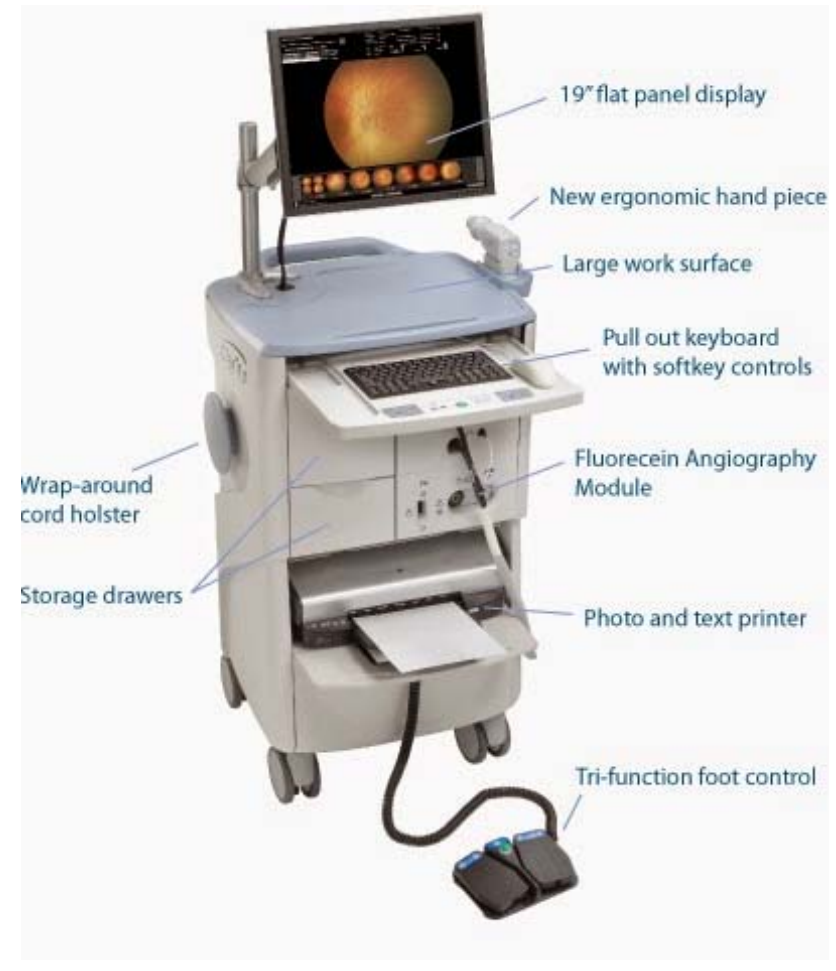

Source: Kubicek et al. (2016c)

\section{System RetCam 3}

The system RetCam 3 is a powerful diagnostic system serving for a spatial imaging of the retinal area especially in children. This system offers lighter and easier adjustable retinal camera with a minimal resolution $640 \times 480 \mathrm{px}$. This system also offers an acquiring the retinal video sequences lasting up to two minutes. There is a possibly of lens changing for achieving an optimal wide-angle retinal image. Physician should select a suitable len with the scale $130^{\circ}$ for the ROP screening, or $120^{\circ}$ which can be used for the examination with a higher contrast but less accuracy. There is also the high contrast len with the scale $80^{\circ}$ suitable for the children and adult patients, and $30^{\circ}$ with a high focusing and resolution for the yellow spot examination or the optical nerve (Imani et al., 2015; Morales et al., 2015).

The system RetCam 3 is primarily intended for the retinopathy diagnostic of the prematurely born infants because it is possible to do screening after two weeks from born (Laaksonen et al., 2016; Xiao et al., 2017). A high risk of the ROP is on newborn with a weight under $1,000 \mathrm{~g}$. By the international standards, each child with a lower weight than 1,500 g should be examined on this disease (Nugroho et al., 2016). RetCam 3 can be utilised also on different retinal diseases, for example on the retinal cancers (retinoblastoma), or other anomalies (Bibiloni et al., 2016; Christodoulidis et al., 2016). 
Figure 4 Retinal images in system RetCam 3 (see online version for colours)

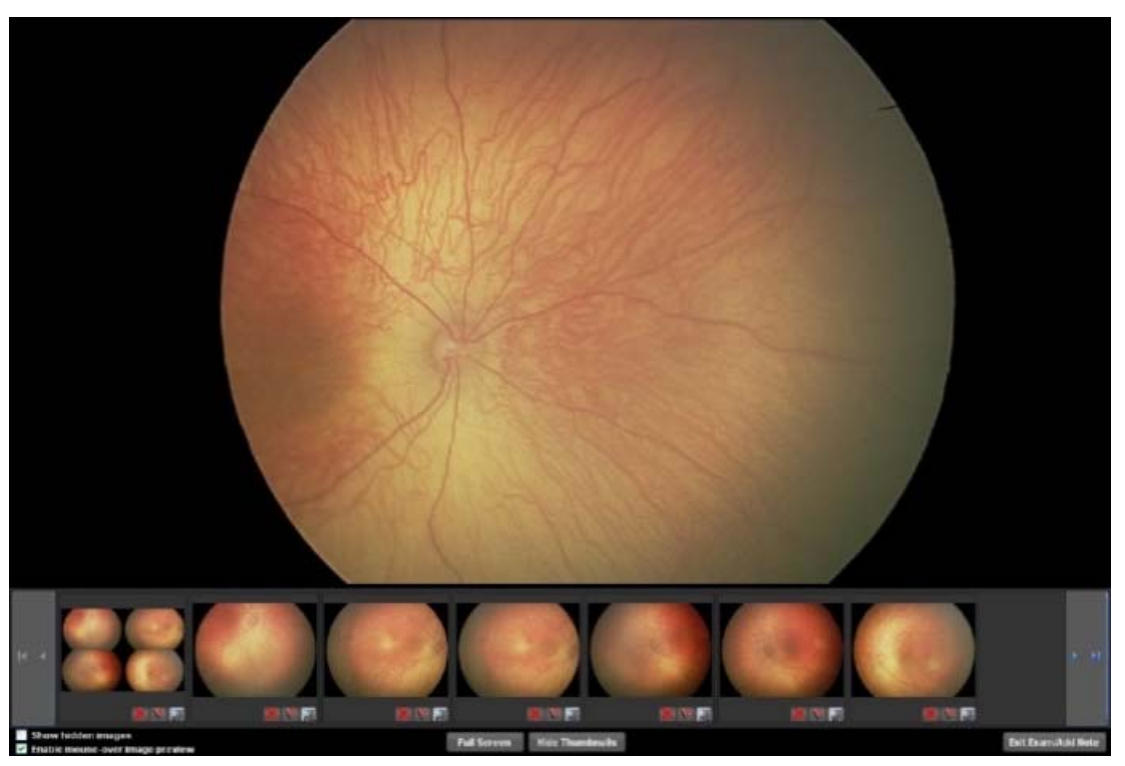

Source: Kubicek et al. (2016c)

\section{Proposed segmentation procedure for retinal lesions and optical disc modelling}

A structure of the segmentation algorithm is adjusted to the retinal image records from the system RetCam 3. If we had processed the retinal images from the fundus camera, we would have worked with high resolution images having a bigger contrast of the optical disc (OD) and the retinal lesions from its background (blood vessels and other retinal structures) (Santhi et al., 2016). In a case of the retinal images acquired from the RetCam 3 system we must keep on a mind that we are working with the low resolution images, on the other hand those retinal images are challenging for segmentation purposes (Kirsali et al., 2014; Awan et al., 2015).

Due to the aforementioned reasons, the proposed segmentation procedure has to include the image preprocessing part optimising the retinal image features of the retinal records. Consequently, the image segmentation procedure is applied. In a context of the segmentation procedure, we suppose that the segmentation procedure is represented by the active snake model. The active snake model represents a time iteration procedure forming the time evolution curve in predefined iteration steps. The main requirement of the clinical practice is to the segmentation algorithm works autonomously without the user intervention. In this regard we have to define the initialisation curve roughly approximating the analysed object (optical nerve and retinal lesions). Consequently, the 
active snake model spreads itself in iteration steps to boundaries of objects which are being segmented. In the consequent step, we obtain the resulting curve reflecting an area of the respective retinal object. This curve is transformed into the binary object. By the binary segmentation we can classify areas of the OD, retinal lesions and image background. Therefore, we can calculate the image area size (geometrical features) of the $\mathrm{OD}$ and retinal lesions in a form of a number of the pixels. As it was stated before, there is a clinical assumption that the OD area should be stable over the time (geometrical features of the OD are unified over the time). Contrarily, the geometrical features of the retinal lesions are not stable, and we suppose the increasing tendency. The main question is how it is possible to objectively measure the retinal lesions (geometrical features) over the time. In the clinical practice, a time evolution of the retinal lesions is only subjectively estimated. It is supposed that this approach is strongly affected by a relative subjective error in a dependence of the clinical ophthalmologic expert skills. Objective feedback brings an objective evaluation to the expert's opinion, and mainly brings diagnosis improving. Also, an important aspect of an automatic time evaluation of the retinal lesions is a prediction model allowing for a time prediction of the retinal lesions development.

\subsection{Preprocessing of retinal images}

This part is intended for achieving as detailed optical nerve and retinal lesions observation as possible. The whole structure of the proposed algorithm is summarised in the Figure 5. Firstly, the image preprocessing is applied due to weaker image features caused by a lower resolution of the RetCam 3. The image processing ensures a better achievement of the retinal image features. This step is done by the edge sharpening, and histogram equalisation ensuring the retinal structures enhancement, it predetermines a higher accuracy of the segmentation. The models of the retinal structures are given by the active contour procedure time-iteratively approximating the OD and retinal lesions areas. The final mathematical models of the retinal structures reflect the size of the retinal lesions against the OD. These features are usable for the retinal lesions time development.

In the input of the entire segmentation procedure we suppose that the retinal image records are generated in the RGB spectrum. In the mathematical sense, it means that the retinal image is composed from three image layers ( $\mathrm{R}$ - red, $\mathrm{G}$ - green and $\mathrm{B}-\mathrm{blue})$. In each of these layers, the retinal structures (OD and retinal lesions) may be imagined under a different contrast, and other image features causing a better or worse retinal structures observation. As far, as it is obvious, that a success of any segmentation procedure is strongly depended on an observation of the native image records. Therefore, we should select an optimal RGB channel where it is supposed as contrast observation of the retinal structures as possible. On a base of the empirical observations we state that the optimal image features are achieved by extracting the $\mathrm{R}+\mathrm{G}$ combination. An extraction of the individual image channels is depicted on the Figure 6 . The best observation $(R+G$ channel) of the OD is depicted on the Figure 7. 
Figure 5 (a) Structure of the proposed algorithm and (b) individual steps of retinal image preprocessing (see online version for colours)

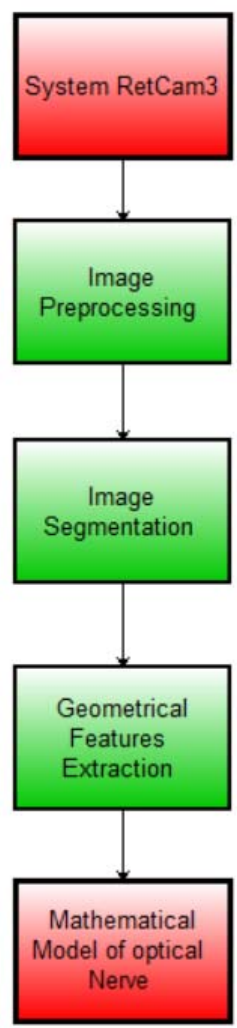

(a)

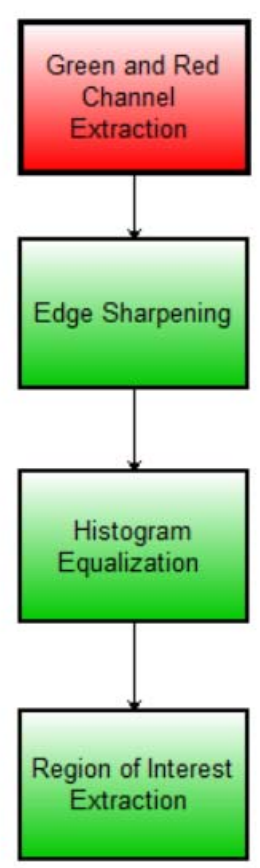

(b)

Figure 6 Comparison of retinal image channels: (a) red channel, (b) green channel and (c) blue channel

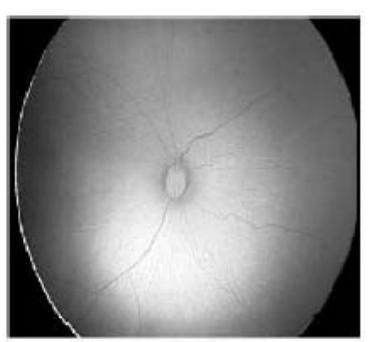

(a)

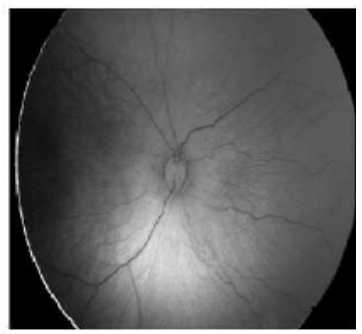

(b)

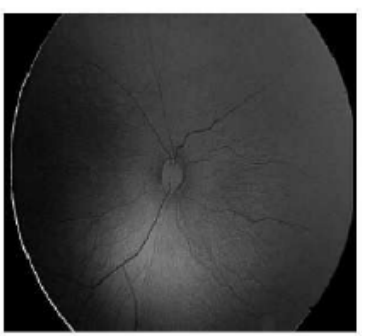

(c) 
Figure 7 Extraction of $\mathrm{R}+\mathrm{G}$ channel combination

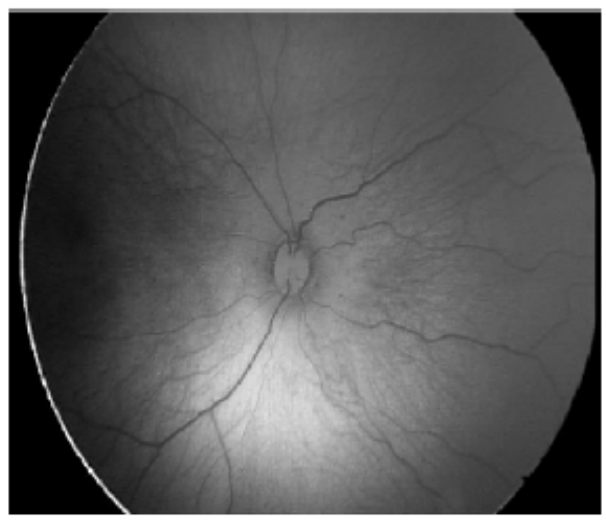

The second part of this initialisation image preprocessing step deals with an extraction of a suitable channel for the retinal lesions. Unfortunately, a quality of the retinal images is not always adequate. In some cases, the retinal images are more or less deteriorated by the image noise and artefacts. Sometimes, physician does not select an optimal location of the retina probe. Consequently, the retinal image may be geometrically distorted, or it is captured under a worse contrast. Different retinal images are indicated on the Figure 8.

Figure 8 Sample of retinal image dataset: different image features on retinal images captured by RetCam 3 (see online version for colours)

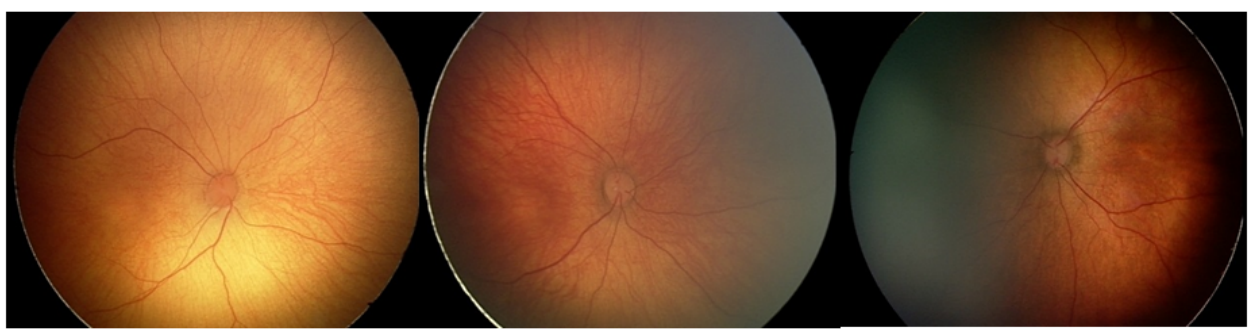

The retinal lesions, which we are focused on in the analysis, are standardly represented by the bloody spots manifesting by the red colour spectrum. A detection of the retinal lesions is more complicated by a fact that its brightness spectrum is frequently identical with the retinal blood vessels. An example of such retinal records is depicted on the Figure 9.

A selection of a suitable RGB layer (Figure 10), or their combination we achieve the retinal lesion features enhancement (brightness enhancement, sharpness, image background suppression). 
Figure 9 Pathologic retinal image containing retinal lesions in a form of the bloody spots (see online version for colours)

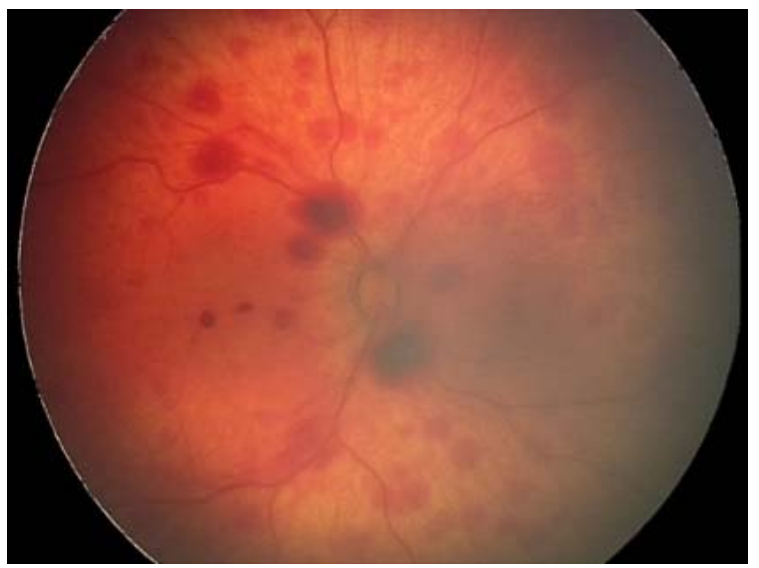

Figure 10 Extraction of individual image layers in retinal image with retinal lesions:

(a) R channel, (b) G channel and (c) B channel (see online version for colours)

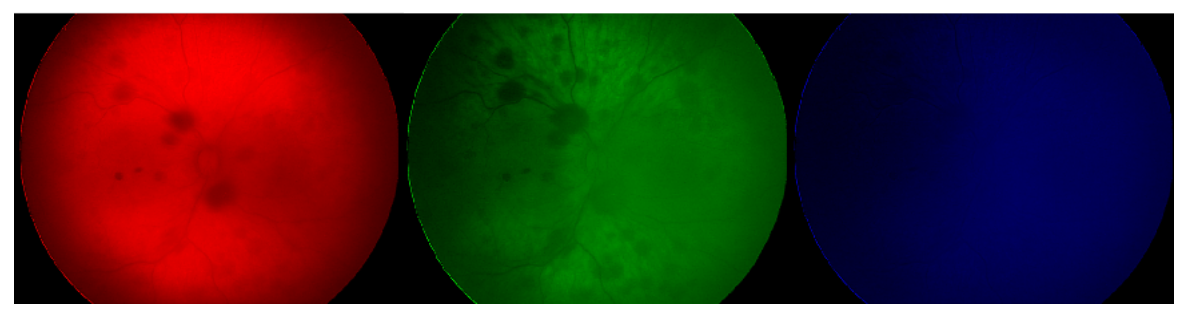

(a)

(b)

(c)

Figure 11 Imaging retinal lesion in $\mathrm{R}+\mathrm{G}$ channel (see online version for colours)

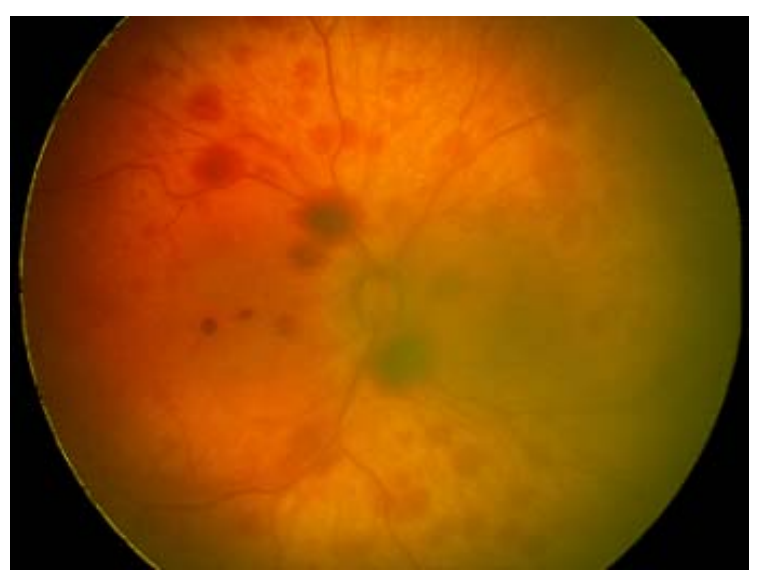


Features of the individual layers are utilised in the algorithm for improving lesions visibility against the retinal background, and result enhancement of consequent retinal preprocessing operations. The best highlighting the retinal lesions is again achieved by the $\mathrm{R}+\mathrm{G}$ combination. During the $\mathrm{R}+\mathrm{G}$ extraction we suppress the blue channel it mathematically means that a matrix representing the blue channel is filled by the zeros. The $\mathrm{R}+\mathrm{G}$ combination in an application on the retinal lesions is indicated on the Figure 11 .

In the next operation, the image sharpening and retinal lesions boundaries enhancement are applied. By those operations, the retinal lesions are highlighted from its background. In a principle of the image sharpening, we increase the retinal lesions contrast along its boundaries where brightness spectrum changes itself. The Boundary is represented by a sufficiently change of the brightness in the retinal image. In the image sharpening, we highlight higher spatial frequencies in the image while other lower frequencies are suppressed.

In the algorithm, we utilise the masking for the image sharpening. This technique is based on a possibility of a subtraction of the blur image from the native image. This procedure utilises a fact that the blurred image contain a higher content of lower frequencies that the original image. By a subtraction we obtain a relative reduction of lower frequencies, and highlighting higher frequencies (retinal lesions boundaries). This process is not obvious on the first sight (Figure 12), however it is an important part for next steps.

Figure 12 (a) Native image and (b) image after masking

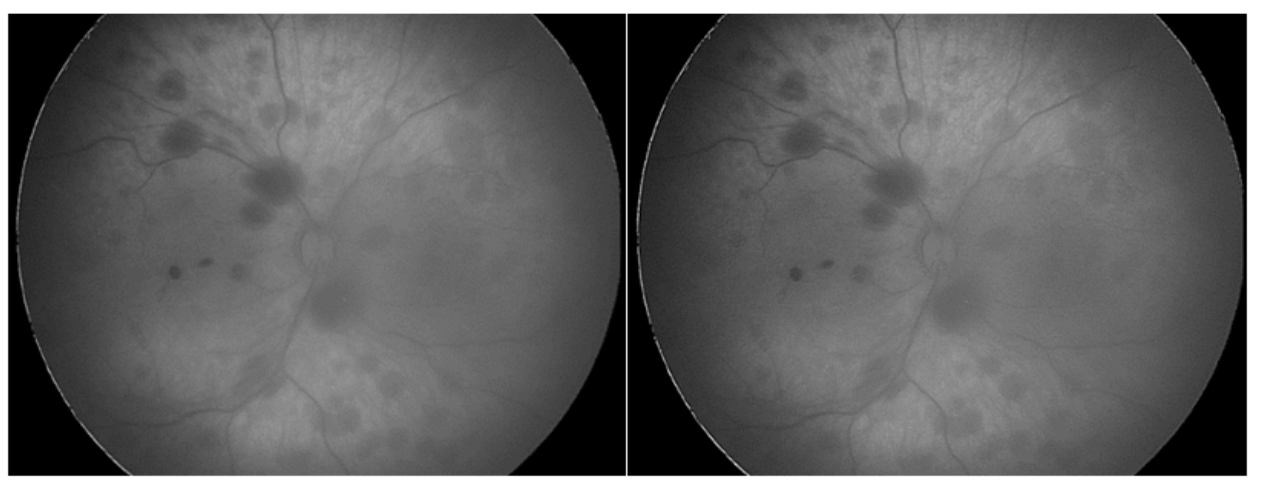

(a)

(b)

Consequently, the image equalisation is carried out. This method is utilised for the image contrast enhancement. This method increases a global contrast in the retinal images. We use this method because the RetCam 3 images are standardly represented by close contrast values. It means that there are not the strong boundaries among the individual retinal lesions and the retinal image background. In a principle, we carry out a change in a brightness level distribution of the image histogram. By the histogram equalisation we obtain an adequate distribution of the individual brightness image levels. 
Figure 13 (a) Native retinal image (b) Histogram (see online version for colours)

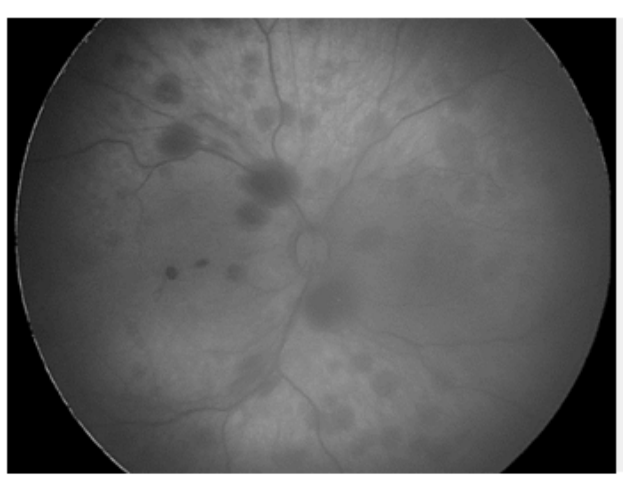

(a)

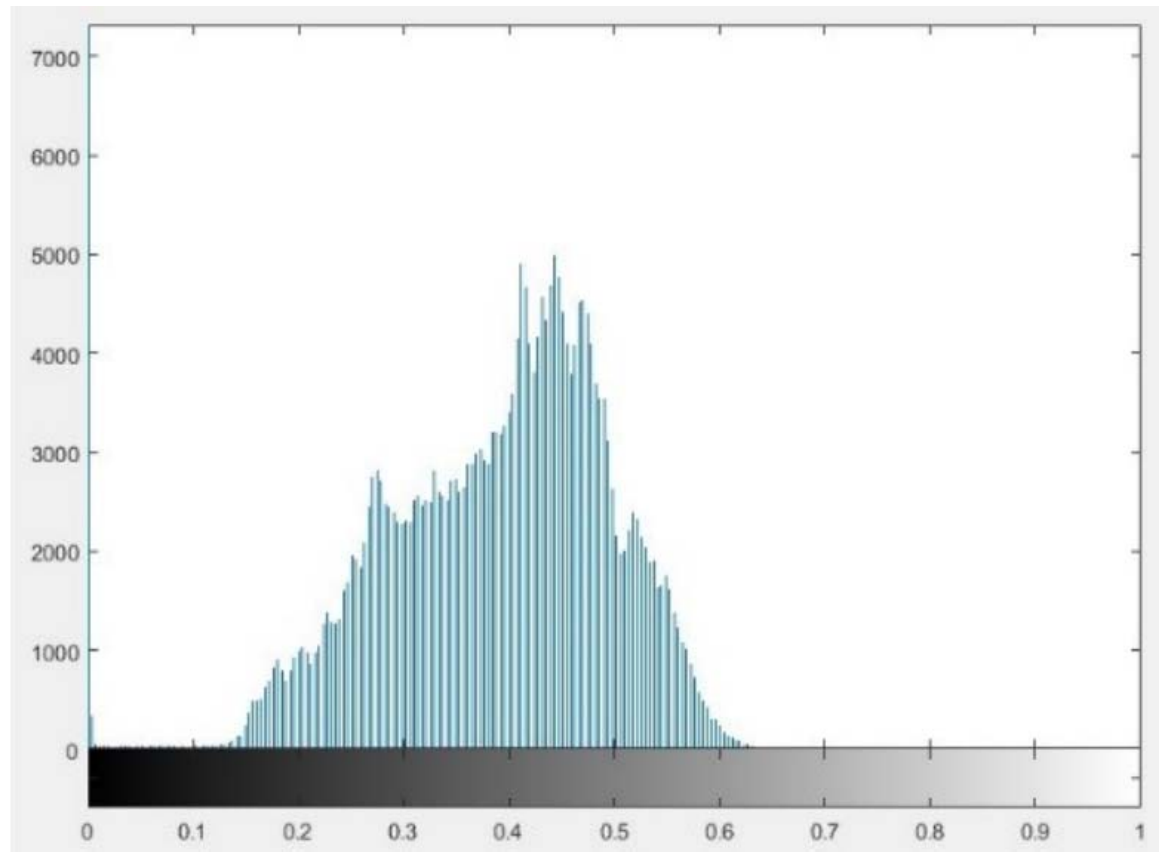

(b)

For achieving better contrast image features of the retinal lesions, the CLAHE method is used (Figure 14). It is a method of the adaptive histogram equalisation (contrast-limited adaptive histogram equalisation). In a comparison with a standard form of the adaptive histogram equalisation, the CLASHE operates on small regions (tiles). It brings a feature ensuring that even small objects are better differentiated under a higher contrast in a comparison with the image equalisation. An advantage is that the method is applied on the smaller image areas against the commonly used histogram equalisation going through the entire image. In each small area of the image, the image contrast is increased step by 
step. The histogram is utilised individually for each image area. The neighbouring areas are consequently jointed by the bilinear interpolation for an elimination of the artificially created boundaries.

Figure 14 (a) Native image and (b) application of CLAHE

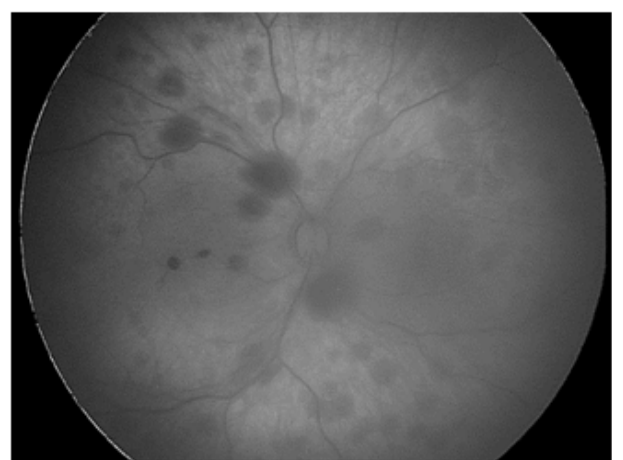

(a)

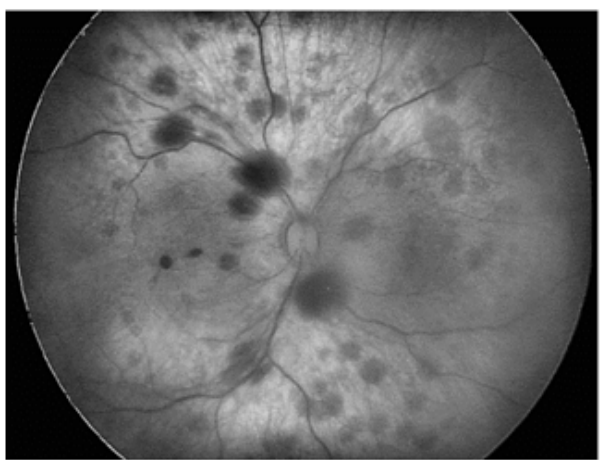

(b)

Figure 15 (a) Original histogram (b) Histogram equalisation (see online version for colours)

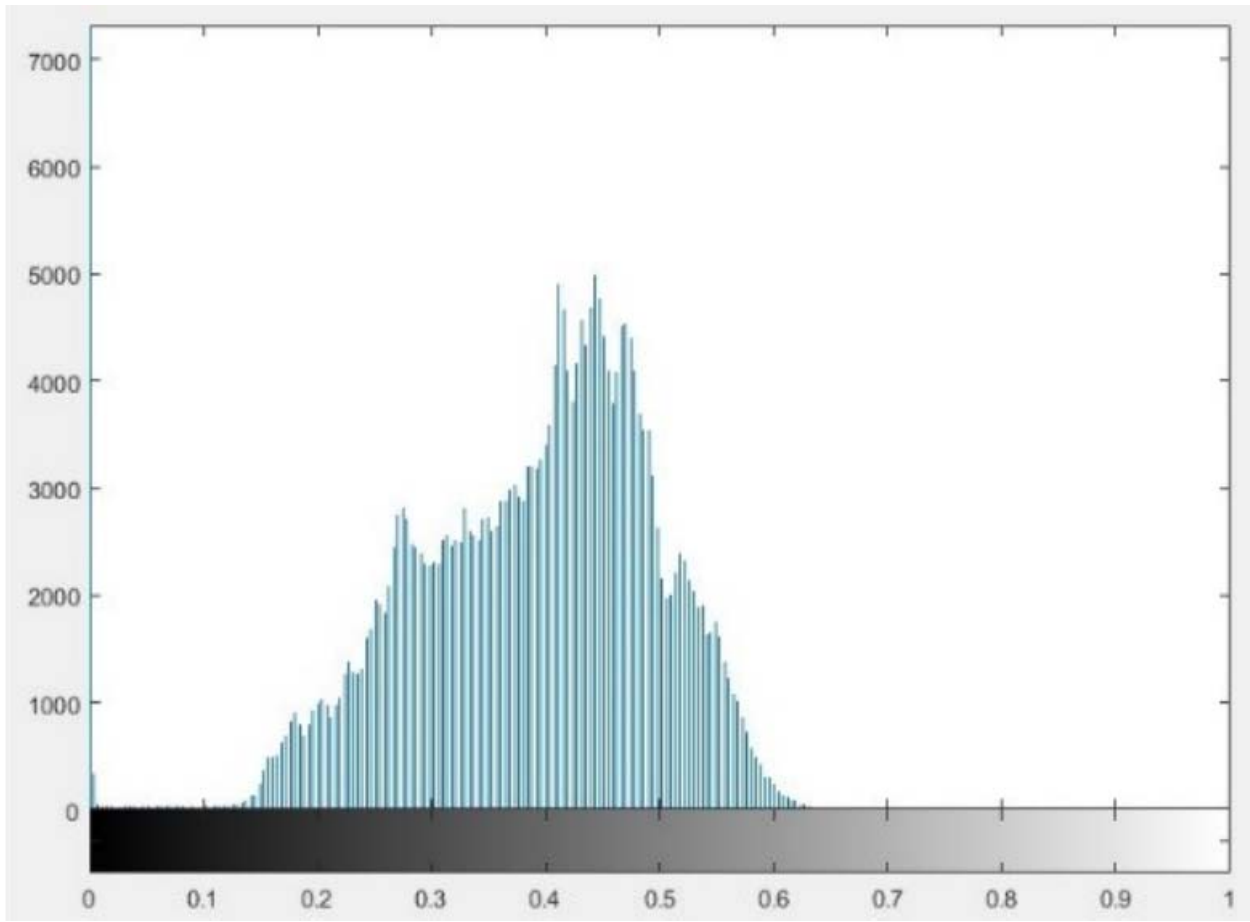

(a) 
Figure 15 (a) Original histogram (b) Histogram equalisation (continued) (see online version for colours)

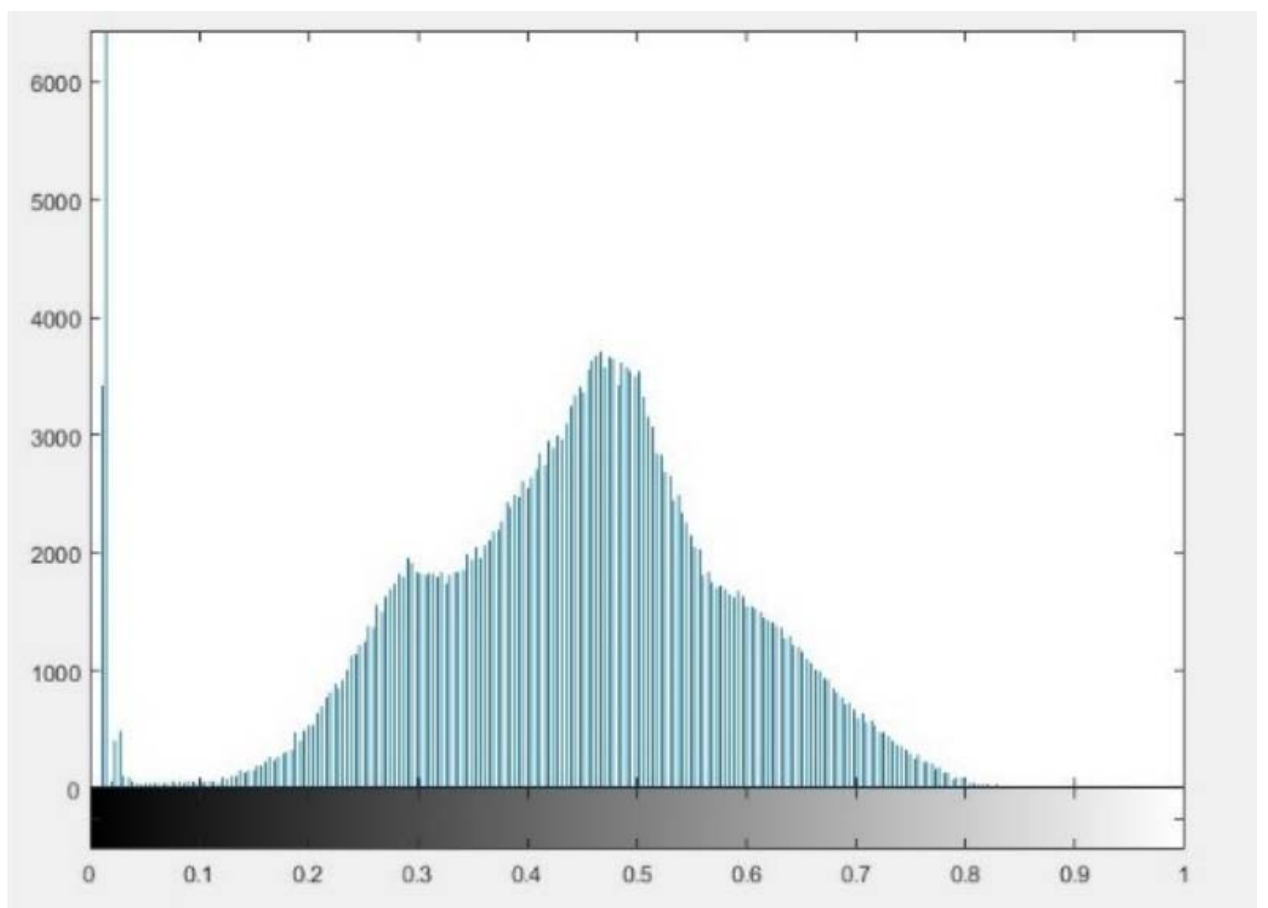

(b)

An important part of the image features enhancement is a brightness transformation (Figure 16). In the proposed algorithm, the brightness transformation is applied in a form of the contrast boosting with a target of a maximisation of the retinal lesion contrast. The process is applied in a form of the point brightness transformation. This operation is applied individually for each pixel. It means that the result of a transformation is calculated on a base of the one pixel.

Figure 16 (a) Native image and (b) point contrast transformation
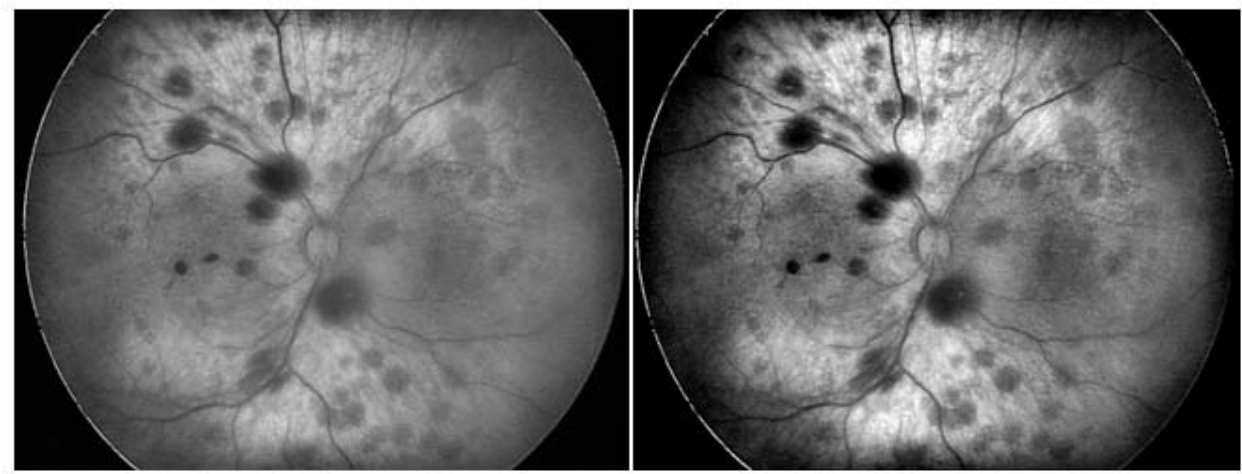

(a)

(b) 
Last step in the preprocessing of the retinal images is a selection of the region of interest (RoI). Regarding to different image quality it was impossible to apply an algorithm on the entire image area, but it is necessary to select a particular area with the respective lesion. This approach brings a benefit in a form of an expansion of the image area. It is obvious that the retinal lesions are pretty small regards to the entire retinal structure. Therefore, by the RoI application, the retinal lesions are expanded to a bigger spatial area (Figure 18). This fact consequently ensures a better visibility of the respective retinal lesions. The RoI is defined iteratively by the user selection (Figure 17). Unfortunately, in the selected area the blood vessels may be present. The retinal blood vessels have an identic brightness spectrum as the retinal lesion therefore it is complicated to eliminate them.

Figure 17 Preprocessed image where area of one retinal lesion is highlighted by RoI (see online version for colours)

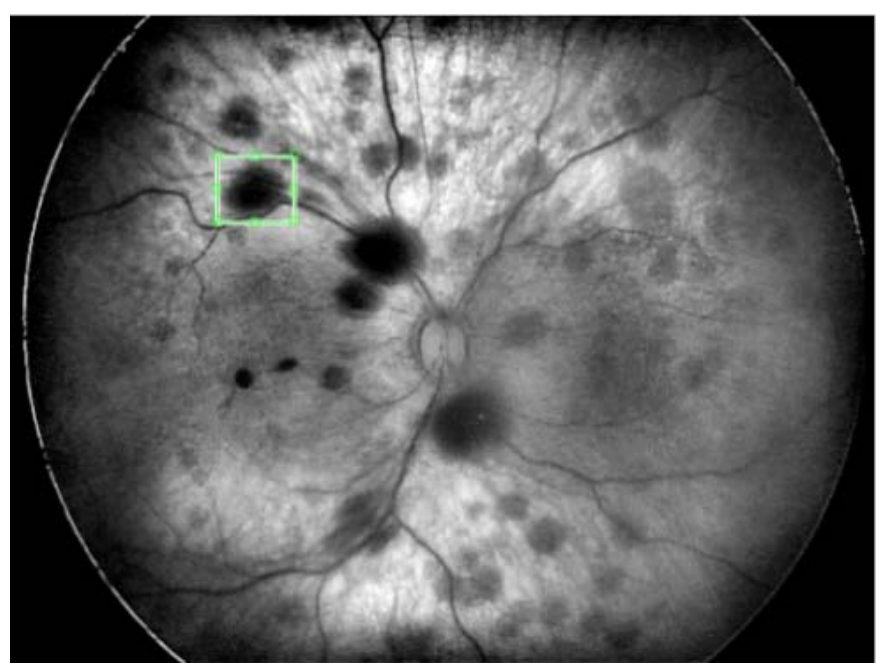

Figure 18 RoI including retinal lesion

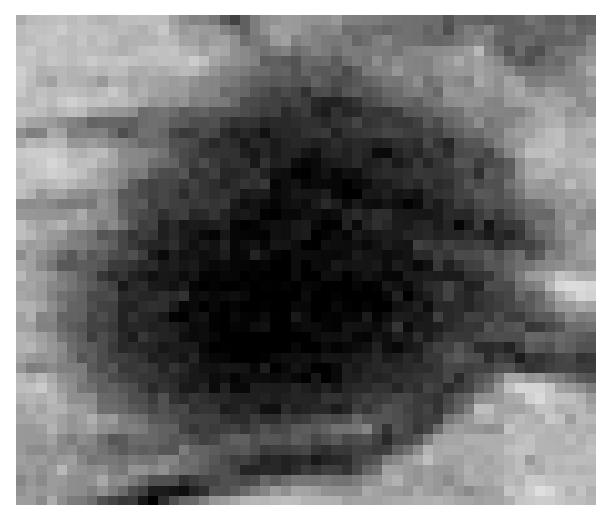




\subsection{Segmentation of retinal images via active snake model}

The segmentation procedure consist the main contribution in the entire retinal lesions modelling. This procedure is able to automatically adjust to the shape of the retinal lesions and the optical nerve to extract models of those structures. From the clinical point of a view, it is the only one appropriate way for reliable extraction features of the optical nerve and the retinal lesions. The optical nerve is approximated by a mathematical model which reflects the geometrical features of the optical nerve area, and allows for a further clinical evaluation in a form of the geometrical features such as the area and the nerve diameter which is commonly clinically called by the abbreviation papilla diameter (PD). The optical nerve can be extracted from the first retinal image (first patient examination), next retinal images acquired over the time give information about a time development of the retinal lesions, and we suppose that the optical nerve has stable geometrical features (OD area).

There are theoretically more alternatives for the retinal structures modelling. One possible way is an employment of the multiregional segmentation splitting the image area into several disjointing parts. Unfortunately, a brightness spectrum of the retinal lesions and the blood vessels are identical. It means that it is impossible to classify the retinal lesions from other retinal structures in a context of the multiregional segmentation. The Active contours (snake) model is able to adopt to the retinal objects in a predefined number of the iteration steps. This presumption represents a good feature for the retinal lesions and the OD modelling via the active snake model. The active snake model is perceived as an iteratively time minimising model which is able to detect specific features in the image area. An important feature is that the snake model represents a flexible model modifying its geometrical features over the time iterations. Regarding a structure of the active snake model, the model is composed from a predefined number of the discrete points (snaxels) connected between each other by straight lines (Figure 19). An evolution of the active contour is performed by an iterative process which controls the final contour shape.

Figure 19 RoI including retinal lesion

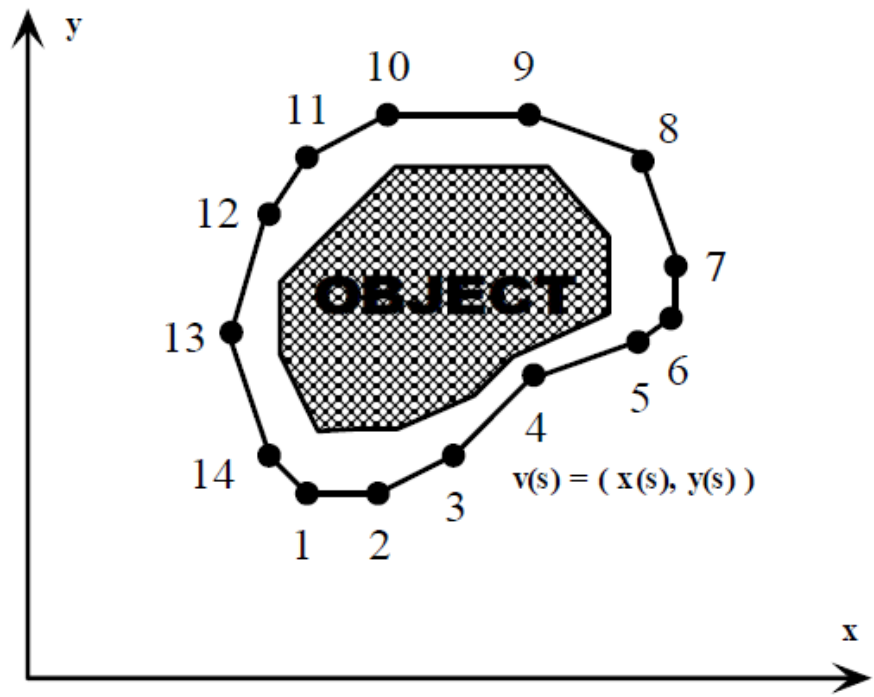


Active snake model is given by sequence of snaxels $s$. This curve can be described by the parametric function (1).

$$
v(s)=[x(s), y(s)]
$$

where $x(s)$ and $y(s)$ represent coordinates $x$ and $y$ through the contour and $s$ signs a normalised index of the control points. The energy function that describes the active contour is composed from two main components, the internal energy and the external energy. Curve compactness is formed by the internal forces. The external forces tend the curve towards to the object's borders.

The internal energy is composed from two parts: the elastic energy and the bending energy. The internal energy is described by (2) and (3).

$$
\begin{aligned}
& E_{\text {int }}=E_{\text {elastic }}+E_{\text {bend }} \\
& E_{\text {int }}=\alpha(s)\left|\frac{d v}{d s}\right|^{2}+\beta(s)\left|\frac{d^{2} v}{d s^{2}}\right|^{2}
\end{aligned}
$$

where $\alpha$ is an adjustable constant that specifies the continuity and $\beta$ is an adjustable constant as well which specifies the contour curving. The elastic and bending energies are defined by the following expressions:

$$
\begin{aligned}
& E_{\text {elastic }}=\int \alpha[v(s)-v(s-1)]^{2} d s \\
& E_{\text {bend }}=\int \beta[v(s-1)-v(s)+v(s+1)]^{2} d s \\
& E_{\text {snake }}^{*}=\int E_{\text {snake }}[v(s)] d s \\
& E_{\text {snake }}^{*}=\int_{0}^{1}\left\{E_{\text {int }}[v(s)]+E_{\text {image }}[v(s)]+E_{\text {con }}[v(s)]\right\} d s
\end{aligned}
$$

where $E_{\text {int }}$ is the internal energy of the curve, $E_{\text {image }}$ is the energy of the image and $E_{\text {con }}$ determines the external limitations (Kubicek et al., 2016a, 2016b).

\section{Mathematical modelling of retinal lesions and OD}

The final mathematical model of respective OD and retinal lesions should reflect the geometrical features of these retinal objects, and reliably approximate an area of a manifestation of these objects in the retinal images (Kubicek et al., 2016c). There are two model representations. Model can be represented by a closed curve reflecting an area of the OD and respective retinal lesions. The main disadvantage of this method is nonreflecting object area it means that we capture boundaries without spatial information reflecting the size of the structure area. Instead of utilising a closed curve, we utilise the binary classification as a final form of the model. The binarisation is a procedure classifying the retinal image into two main regions.

Firstly, the boundaries of the retinal objects are approximated by the closed smooth curves. In the initialisation phase the circle is defined. This circle is iteratively deformed 
within predefined iterations. The following figures represent an evolution of the active contour model for three retinal lesions and the OD. On the retinal lesions we present a comparison of 100 and 200 iterations (Figure 20), contrarily the OD area approximation is presented for 100, 150, 200 and 300 iterations (Figure 21).

Figure 20 An active contour segmentation of three retinal lesions from RetCam 3: (a) initial contour, (b) 100 iterations and (c) 200 iterations (see online version for colours)
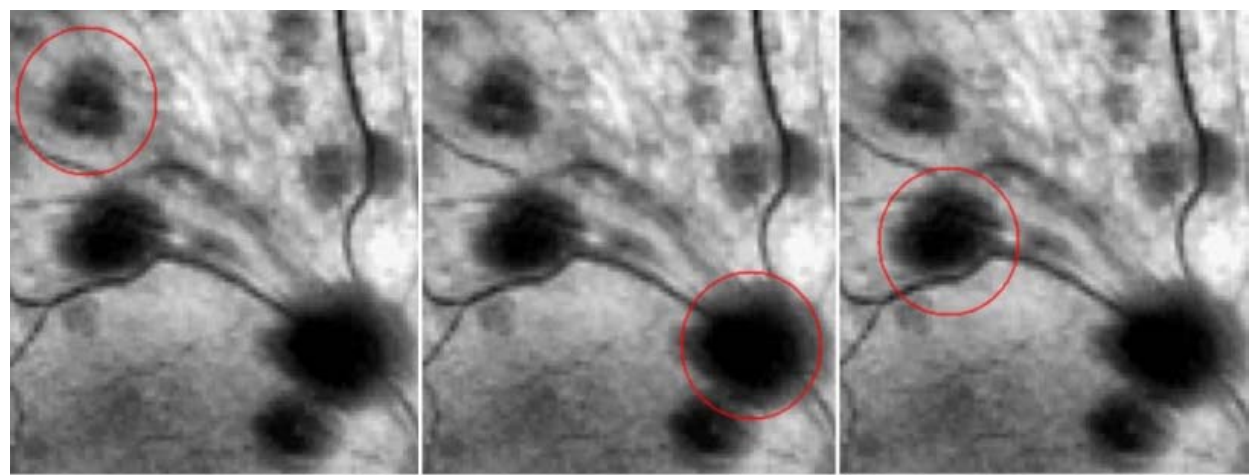

(a)
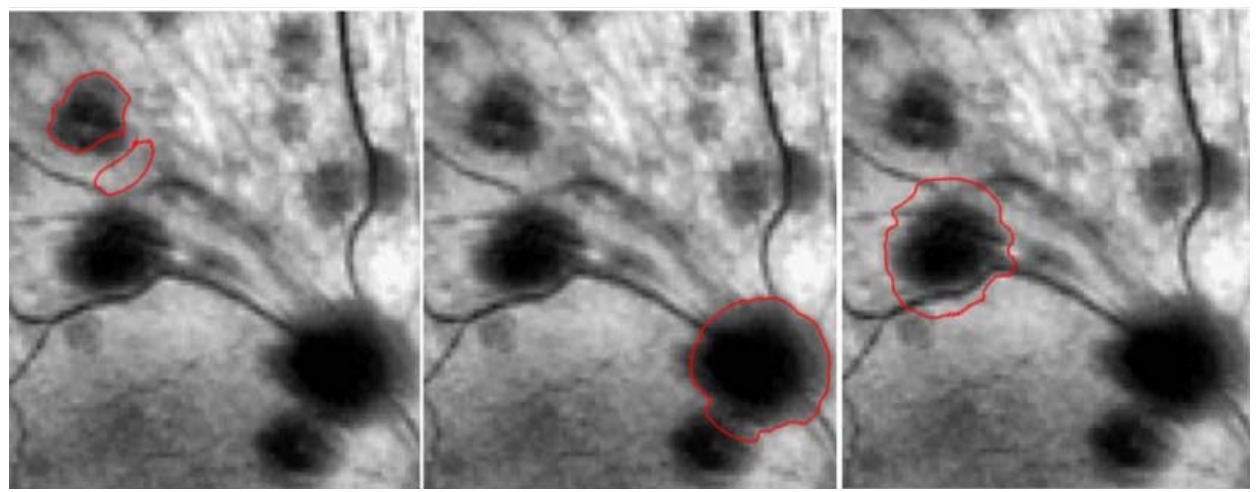

(b)
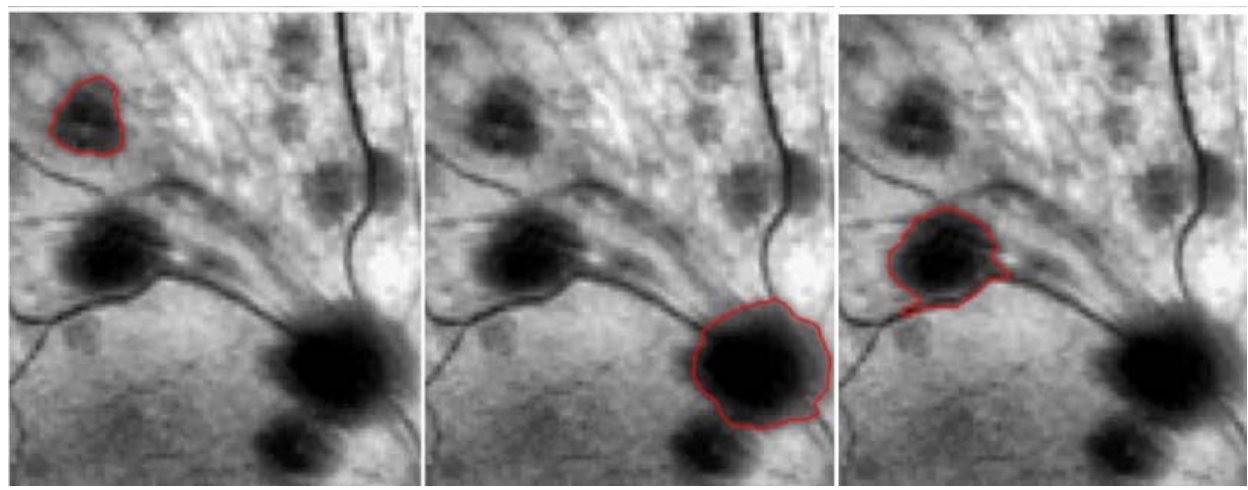

(c) 
Figure 21 An active contour segmentation of OD from RetCam 3 (see online version for colours)

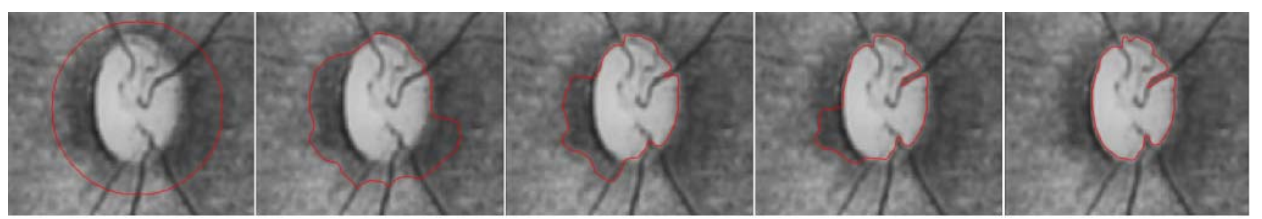

Note: From left: initial contour, 100, 150, 200, 300 iterations.

\subsection{Model of $O D$}

Before applying the segmentation procedure, the initial contour is placed on the segmented object. There is a requirement so that the initial mask reliably approximates geometrical features of the OD and the lesions. There are two reasons for this task. At first, an area of similarity has significant impact on a number of the iterations. If we used an initial contour badly approximating the retinal structure (geometrical features of contour are significantly different) we would have to use a bigger number of the iteration steps. This fact causes an increased computational time. An area similarity is important due to an accuracy of the segmentation. If the initial curve is underestimated or overestimated the segmentation snake has tendency goes out of the object of interest. This fact would lead to an improper segmentation results, and a distortion of a final model of the retinal structures. A placement of the initial contour on the OD area is depicted on the Figure 22. Besides an area of the initial contour, a key feature is also the geometrical shape. The geometrical shape also determines segmentation accuracy. The best way is a utilising the geometrical shape which is similar to analysed objects. In a case of segmenting the optical nerve and the retinal lesions, the best compromise represent either the ellipse or the circle because those retinal objects exhibit circular geometrical features closely similar to the circle and ellipse.

Figure 22 (a) Placement of initial contour roughly approximating area of OD (b) Binary mask of defined contour (see online version for colours)

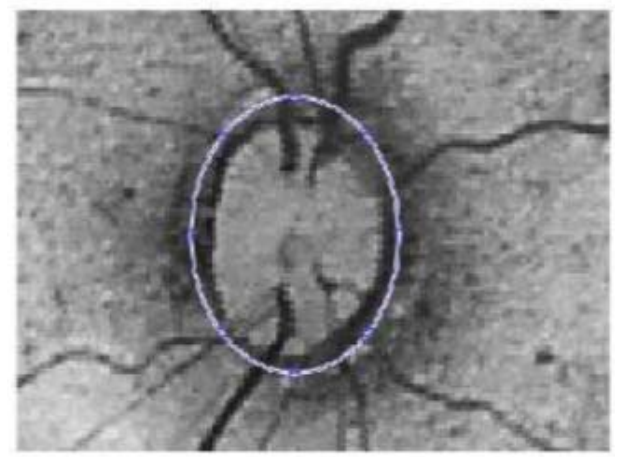

(a)

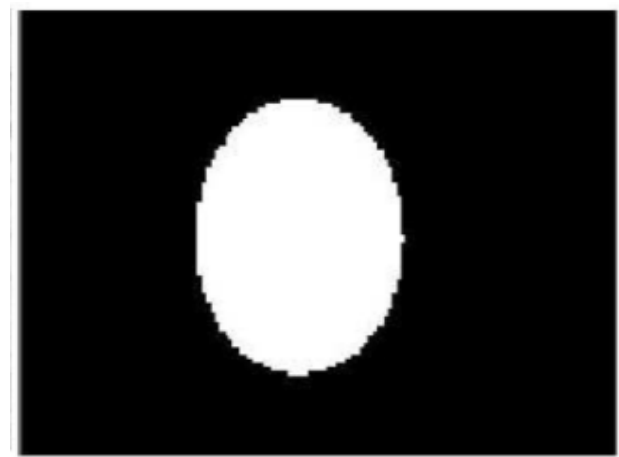

(b)

The final model of the optical nerve is formed by a time evolution of the initialisation contour. Final shape is especially affected by a number of the iterations. On a base of the 
experiments using of 300 iterations appears itself as the best compromise for reaching as relevant approximation of the optical nerve as possible (Figure 23). If we underestimate the segmentation process in a context of a number of the segmentations, the segmentation curve badly approximate a target area. Other words speaking the segmentation curve does not manage to achieve boundaries of the optical nerve. On the other hand if we overestimate the segmentation process by an excessive number of the iterations, the segmentation curve may have tendency to spread itself out of the segmented area. Consequently, a modelled area of the OD will be much bigger than an original area of the OD. The snake method has a one substantial advantage it allows for to track individual steps of the segmentation process. By this positive feature, the segmentation curve can be terminated earlier than in the final step of the segmentation. An evolution process of the segmentation procedure is depicted on the Figure 23.

Figure 23 The time evolution of the active contour from (1) initialisation contour to the (6) final model of optical nerve

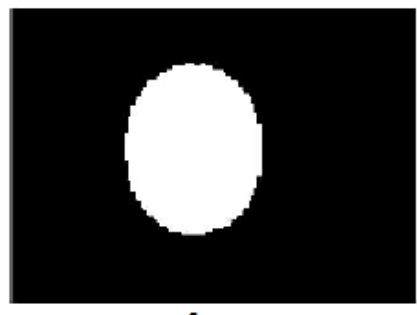

1

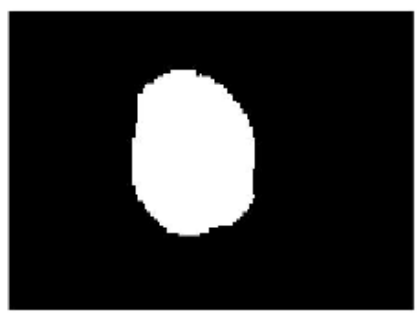

4

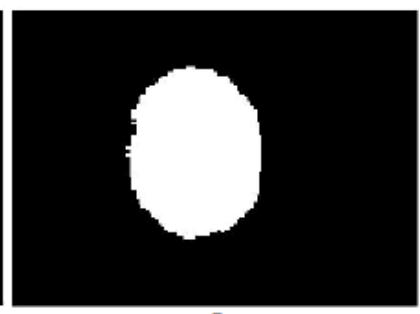

2

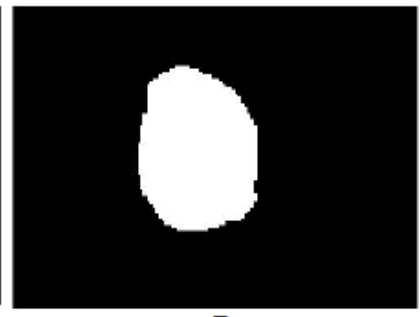

5

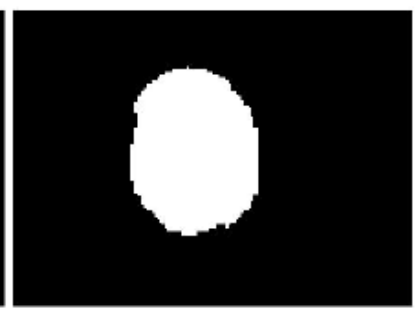

3

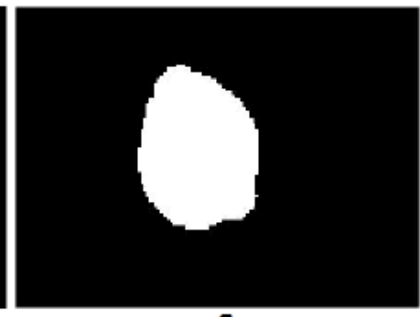

6

An important part of the segmentation model is defining the final area (binary model) of the OD. During the segmentation, the active snake model modifies the internal and external energy. In this context, we can interactively determine the energy map which is related to each segmentation step. In the energy map, we can recognise two essential areas characterising the energy distribution (Figure 24). An area inside of the segmentation curve have negative values of the energy, contrarily an area outside of the segmentation curve have positive values of the snake energy. By this selective approach we can classify whether a respective pixel belongs inside or outside of the OD.

An important part of any segmentation algorithm is verification. We need to have a certain feedback about the algorithm effectivity. For needs of the clinical ophthalmologists, suitable solution is the image fusion. It is supposed that the original native image area is overlaid by the segmentation result. By this approach we can visually track a correlation of the OD model with the native record where the OD is present. Extract of the image fusion is depicted on the Figure 25. 
Figure 24 (a) Energy map of active snake model applied on optical nerve and

(b) binary classification of OD area (see online version for colours)

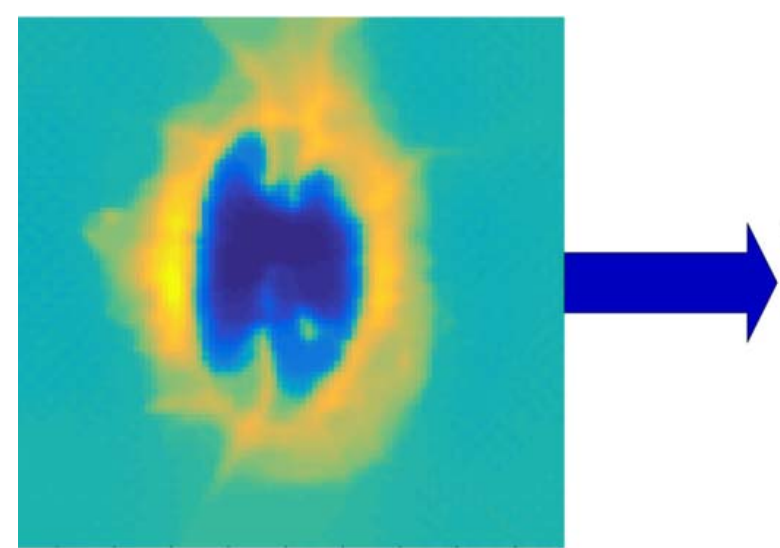

(a)

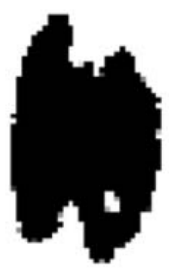

(b)

Figure 25 Testing image fusion algorithm on the image data extract

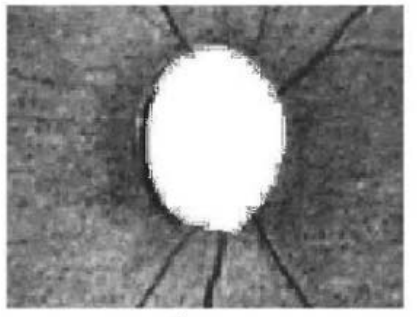

1.

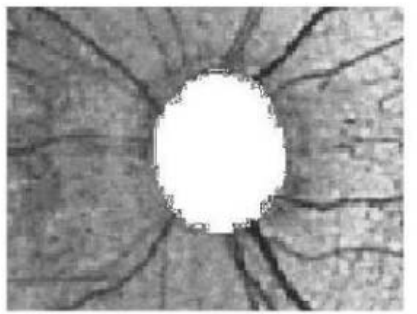

4.

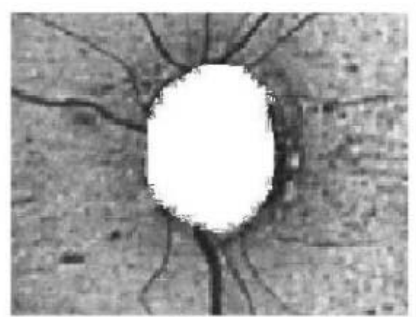

2.

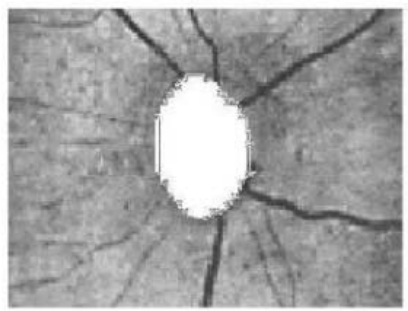

5.

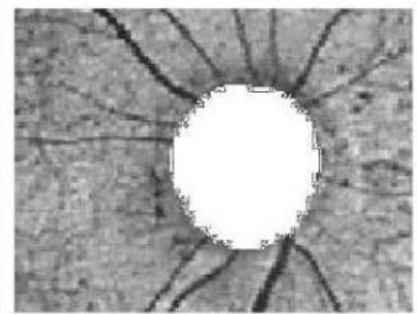

3.

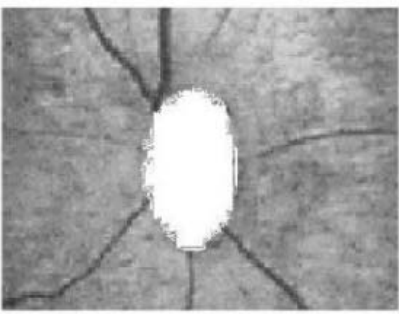

6.

\subsection{OD geometrical features extraction}

The last step of the OD segmentation is the geometrical features extraction. There are several methods which are able to transform the image distance on real measurements units. Unfortunately, we are limited by a fact that the real distances acquired by the system RetCam 3 are unknown. It means that a distance on the image does not reflect the real retinal distance. Considering this fact, the OD parameters are measured and evaluated in the image units (pixels). In our method, the OD area is approximated by the circle with a same area as the original binary model. Therefore, the most important the 
OD parameters are area and OD diameter. Those parameters are especially important for further processing of the retinal lesions where the OD is given as a reference point. In the processing of the retinal lesions we consider that the lesion area is measured against the OD radius.

On a base of the empirical studies we know that the OD can be reliably approximated by the ellipse where the radius diameter of major and minor axes is approximately 0.14 $\mathrm{mm}$. It means that the OD is closely resembled to the circle. On a base of this fact, the OD is approximated by the circle with a same area as the original binary record. The OD radius is consequently calculated by (8) and (9).

$$
\begin{aligned}
& S=\pi \cdot r^{2} \\
& r=\sqrt{\frac{s}{\pi}}
\end{aligned}
$$

where $S$ indicates an area of the OD and $\mathrm{r}$ indicates a radius of the OD in pixels. We can allow this poor precision caused by an approximation because the OD and the retinal lesions are nearly identical with the circle. Therefore, the circle approximation appears as a suitable solution. The resulting comparison of its radiuses is in accordance except for a negligible deviation.

Lastly, we have tested a recurrent application of the segmentation procedure on the real native retinal records. We pay attention to the biggest difference of the OD radius during the recurrent detection.

We have done the OD modelling on a sample of the 120 patient records. It hardly ever happened that the segmentation results exhibited significantly different results. In majority of cases we registered an identical detection results. In the 51 cases we registered totally identical detection ( $42 \%$ of test). The biggest difference is equalled to the three pixels, while we can predict that this difference is caused by an inappropriate placement of the initial contour. Important fact is also an average error per one case which is 0.73 pixels. If we take under consideration that a spatial area of the retinal record is 307200 pixels, an average detection fail is approximately $0.0002 \%$. The following chart represents an extract of the algorithm testing. For each image, the segmentation procedure has been tested three times. Error indicates the biggest difference between individual segmentation attempts.

\begin{tabular}{|c|c|c|c|c|}
\hline Image & 1 Segmentation & 2 Segmentation & 3 Segmentation & Error \\
\hline 1 & 23 & 22 & 23 & 1 \\
\hline 2 & 23 & 22 & 22 & 1 \\
\hline 3 & 20 & 20 & 21 & 1 \\
\hline 4 & 21 & 22 & 21 & 1 \\
\hline 5 & 17 & 17 & 17 & 0 \\
\hline 6 & 17 & 18 & 17 & 1 \\
\hline 7 & 18 & 17 & 17 & 1 \\
\hline 8 & 23 & 23 & 23 & 0 \\
\hline 9 & 19 & 19 & 17 & 2 \\
\hline 10 & 20 & 20 & 20 & 0 \\
\hline
\end{tabular}

Table 1 Testing of recurrent application of segmentation 


\subsection{Retinal lesions modelling}

A second crucial part of the segmentation procedure is a modelling of the retinal lesions. In a comparison with the OD, we suppose that the retinal lesions are not stable over the time, while from the clinical point of a view, the OD has same geometrical parameters over the time. A disadvantage of the retinal lesions modelling is an inability of measures the real geometrical parameters of the retinal structures. The system RetCam 3 allows for only an export of the native retinal images without any tool allowing for the measurement. Therefore, all the geometrical parameters are commonly calculated in the pixels. From the clinical point of a view we evaluate an area of the respective retinal lesion in a dependence of the OD. Other words speaking, an evaluation of the retinal lesions says how many times the OD fits itself inside of the retinal lesion. This parameter is determining for the clinical evaluation of the retinal lesions over the time.

For extraction of the retinal lesions in a form of the binary model we utilise a same image preprocessing procedure as in a case of the OD. We tackled with an issue of an insufficient contrast of the lesions against the image background. After applying the contrast transformation (concluding step of the image preprocessing) we obtain a final image for the segmentation (Figure 26).

Figure 26 Sample of two testing images after image preprocessing

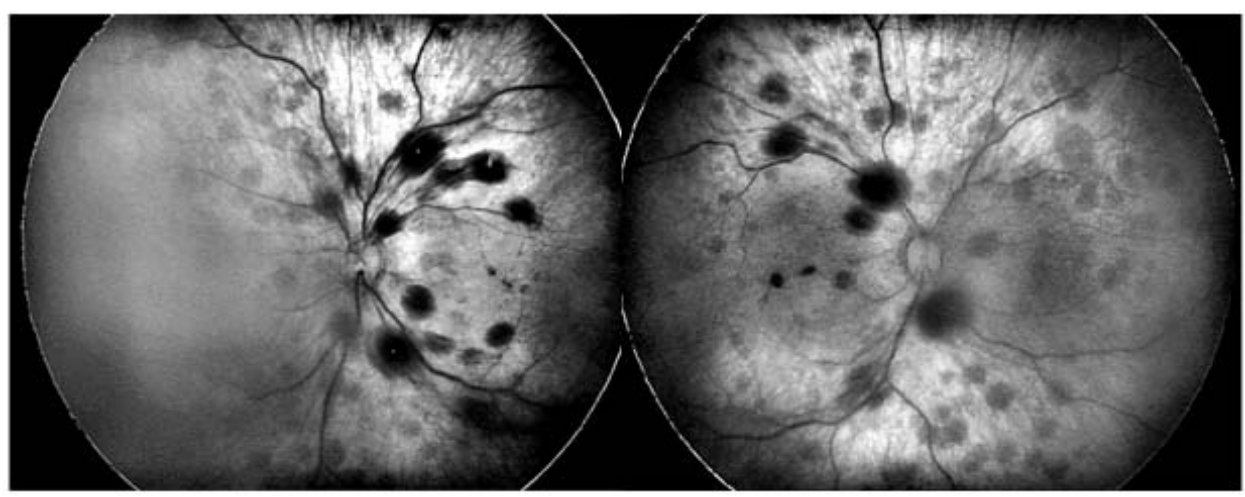

The main contribution in the retinal lesions modelling is the segmentation of the individual retinal lesions. We have tackled with a same issue as if in a case of the OD. Individual retinal lesions are relatively small against the entire retinal image area. Therefore, it is needed to expand an area of the retinal lesions with a target of a better visibility of the retinal lesion, and an accurate effect of the segmentation algorithm. This task is connected with the interpolation procedure increasing a number of the pixels in the RoI, and enhances the contrast features.

The segmentation procedure is applied on the RoI where the individual retinal lesion is present. After the segmentation is done, a final model of the retinal lesion is done by the binary classification. The following outputs represent the segmentation results of the individual retinal lesions. The lesions have a miscellaneous characteristic in its placement, and also in its shape. There are the lesions which are completely divided from each other, and therefore they are simply detectable. On the other hand, there are also the lesions laying on the retinal blood vessel, or closely to them. First result of the 
segmentation is depicted on the Figure 27. A model of the individual retinal lesion is generated on a base of the 300 iterations.

Figure 27 Testing the retinal lesions segmentation for six dominant retinal lesions (see online version for colours)

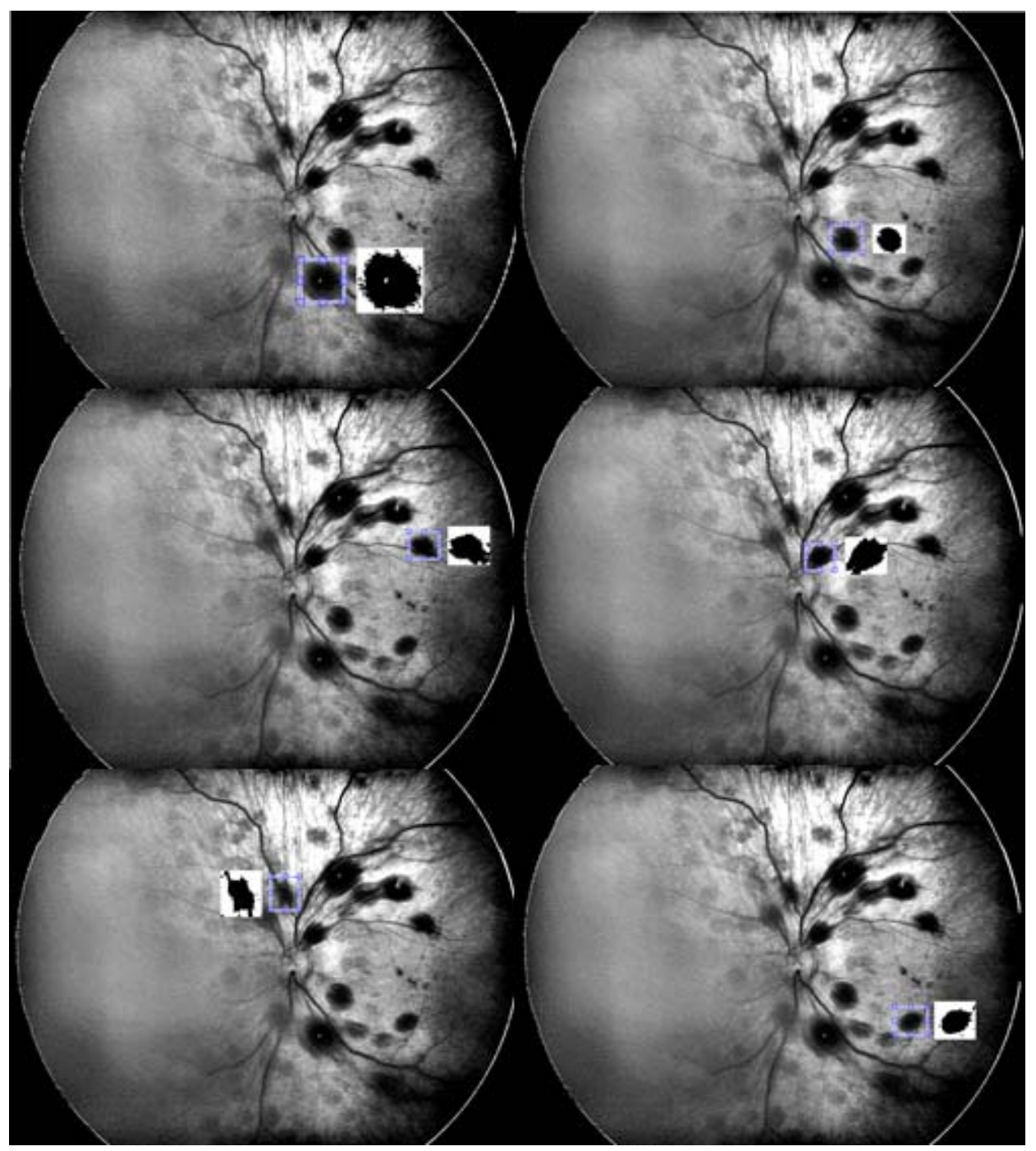

Note: 300 iterations are used for segmentation.

Second testing image does not dominate such he dark lesions as the first testing image (Figure 27). Nevertheless, the segmentation algorithm is able to detect even those less contrast retinal lesions (Figure 28). 
Figure 28 Testing the retinal lesions segmentation for six dominant retinal lesions (see online version for colours)

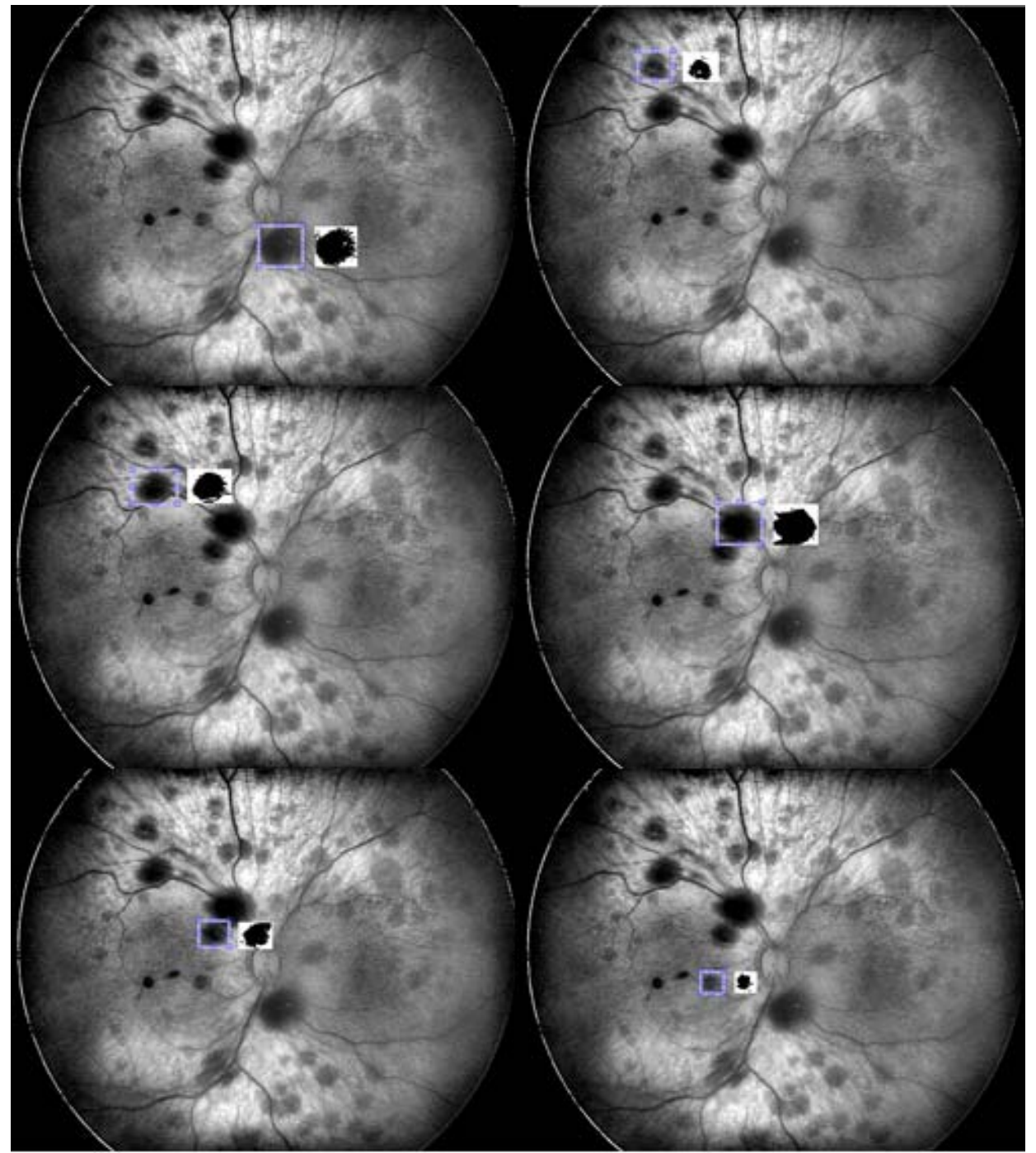

Note: 300 iterations are used for segmentation.

The following retinal image (Figure 29) shows one of precisely detectable lesions. The lesion is circumscribed well, and fortunately there are not any other retinal objects on the extracted RoI.

Since that a position of the retinal lesions is not always such ideal like in the previous case where the lesion was placed individually, in some cases there is a distortion by other objects which cannot be eliminated from the retinal image. In the following image 
(Figure 30), there is the retinal lesion which we cannot precisely extract from the image background. The retinal lesion model is distorted by the adjacent blood vessel crossing the retinal lesion. The resulting retinal lesion radius and area is overestimated by the blood vessel pixels.

Figure 29 Particular imaging of retinal lesion model (see online version for colours)

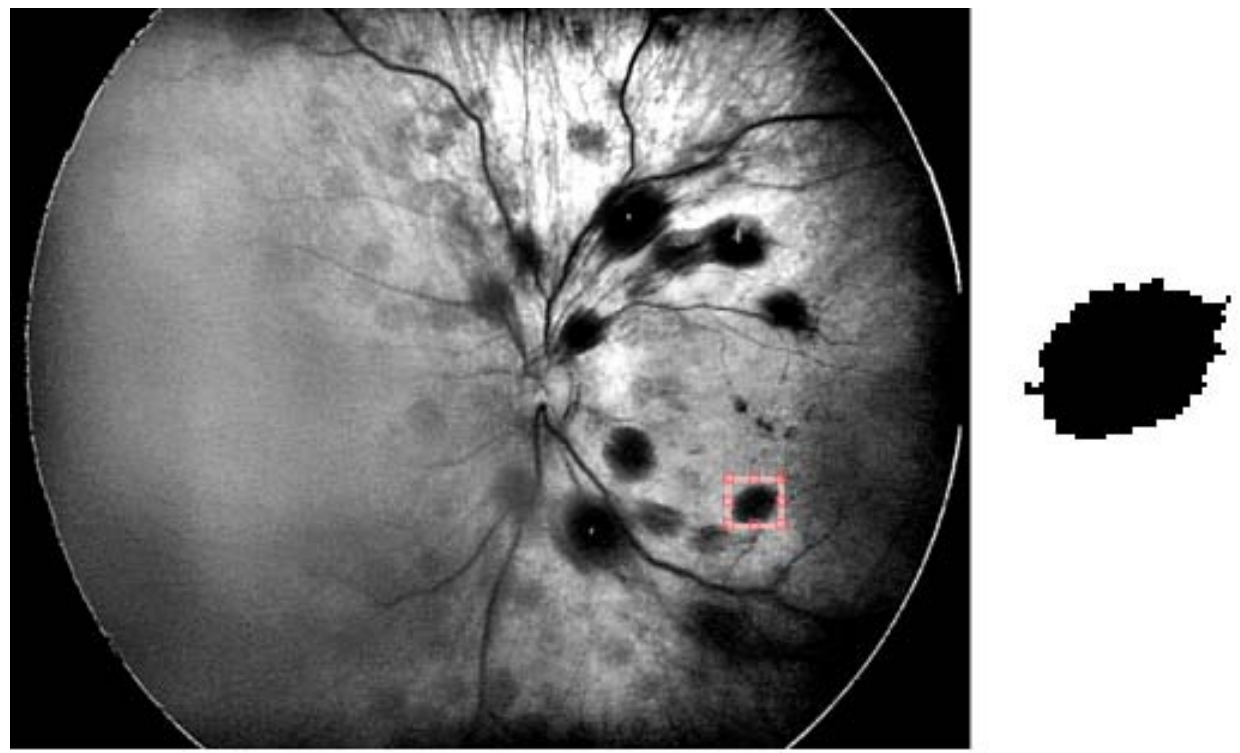

Figure 30 Retinal lesion model with imprecise classification caused by adjacent retinal blood vessel (see online version for colours)
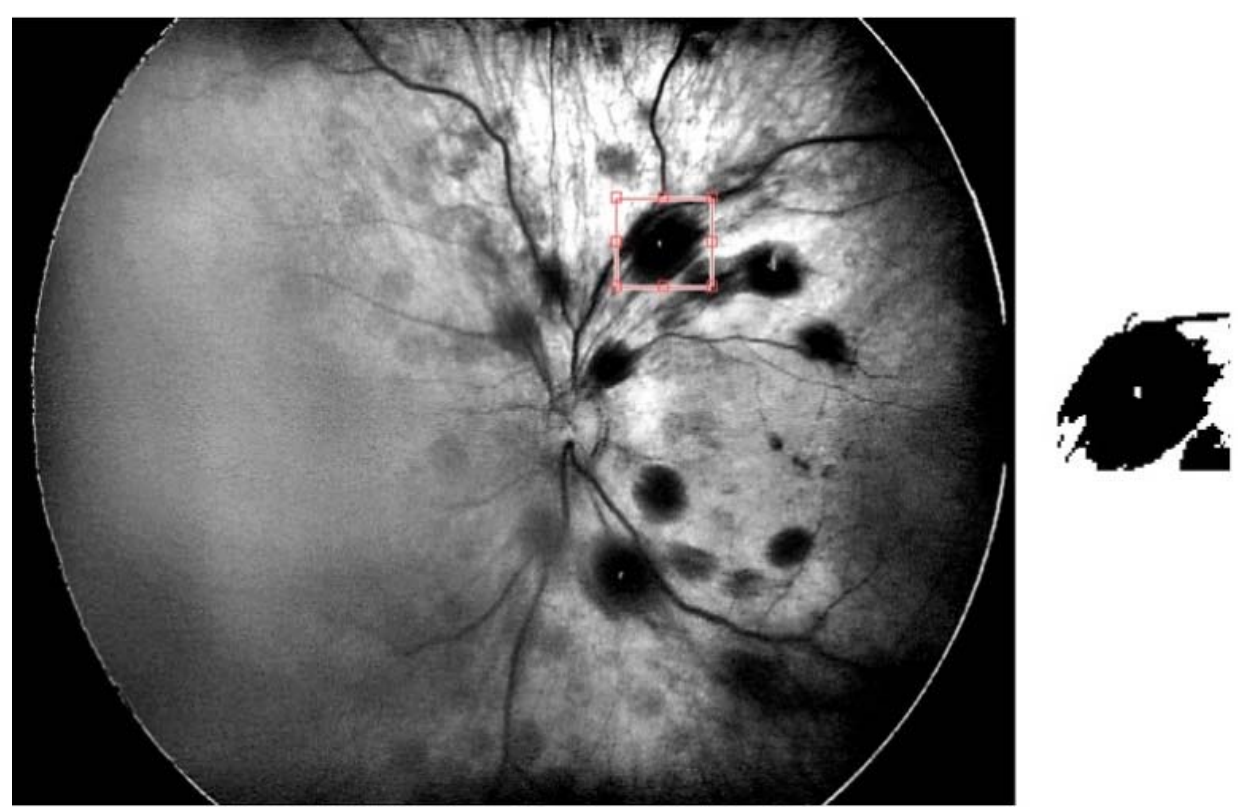


\subsection{Extraction of retinal lesions geometrical features}

The retinal lesions are characterised by its radius and area. A radius and area of the lesion depends on the lesion location, and a presence of unwanted objects as the retinal blood vessels, or other tiny adjacent lesions which cannot be eliminated from the RoI. If the RoI consists other objects with a similar brightness spectrum as the retinal lesions, the active snake model might have tendency to spread to this area. In a result, the final model would be overestimated by these objects. A reliability of the method is partially depended on the other objects jointed with the respective retinal lesion.

The lesion area is compared with an area of the OD. The results are shown in Table 2. After the OD and retinal lesions segmentation procedures, its areas are calculated in a form of a number of the pixels lying inside those retinal objects. The following outputs (Figure 31 and Figure 32) show results of the retinal lesion models for the geometrical parameters extraction.

Figure 31 Segmentation model for geometrical parameters extraction for testing image 1 (see online version for colours)
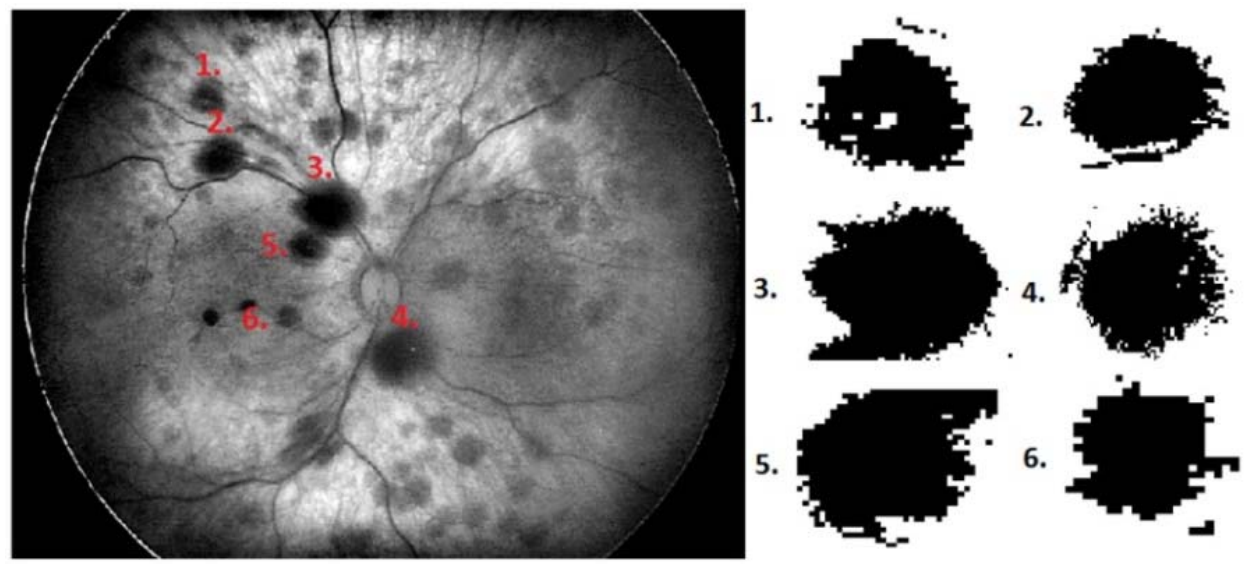

Figure 32 Segmentation model for geometrical parameters extraction for testing image 2 (see online version for colours)

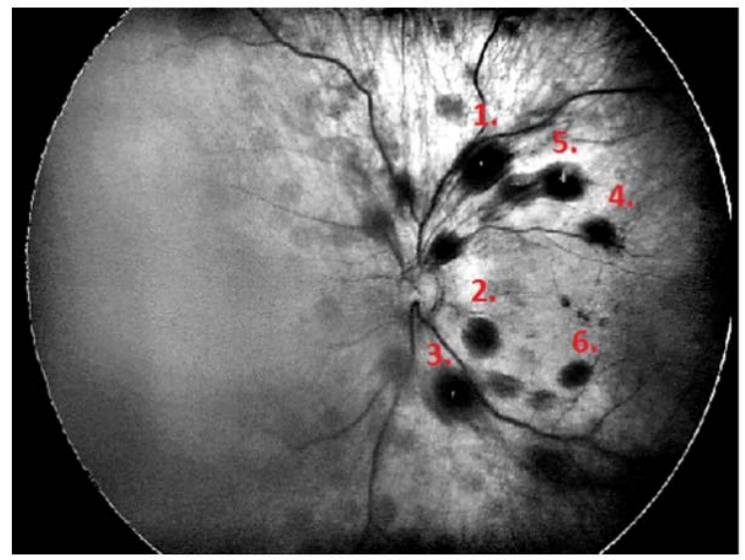

1.

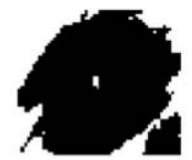

.

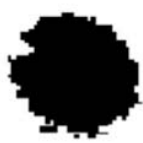

3.
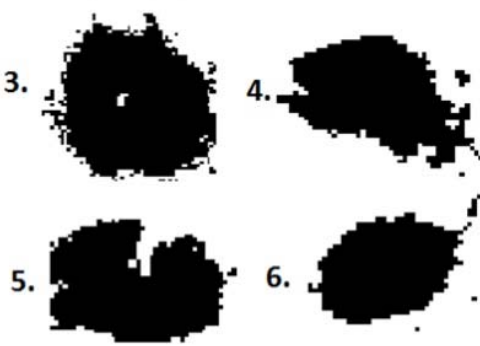

6.

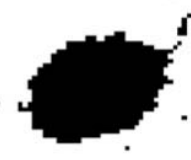


Table 2 Retinal lesions geometrical features extraction for testing image 1

\begin{tabular}{lcccc}
\hline Lesion ID & Lesion area & Lesion radius & \% area of OD & OD area \\
\hline 1 & 503 & 13 & 44 & 1,139 \\
2 & 1,139 & 19 & 100 & \\
3 & 1,915 & 25 & 168 & \\
4 & 1,752 & 24 & 154 & \\
5 & 739 & 15 & 65 & \\
6 & 254 & 9 & 22 & \\
\hline
\end{tabular}

Table 3 Retinal lesions geometrical features extraction for testing image 2

\begin{tabular}{lcccc}
\hline Lesion ID & Lesion area & Lesion radius & \% area of OD & OD area \\
\hline 1 & 1,977 & 25 & 194 & 1,018 \\
2 & 723 & 15 & 71 & \\
3 & 1,908 & 25 & 187 & \\
4 & 757 & 16 & 74 & \\
5 & 916 & 17 & 90 & \\
6 & 546 & 13 & 54 & \\
\hline
\end{tabular}

On a base of the results, that the six analysed lesions take less than $100 \%$ OD area. Five tested lesions take more than $100 \%$ of the OD, and one lesion is identical as the OD.

\subsection{Quantitative comparison of retinal modelling}

In the last step, a quantitative comparison of the active contour model applied on the RetCam 3 data is carried out. A quantitative comparison is carried out on a sample of the 30 RetCam 3 records. As ground truth, the manual segmentation of the retinal lesions and the OD is utilised in a form of the retinal object size. In each case, the manual segmentation has been performed three times to eliminate subjective error. Finally, an average value from three manual segmentations is considered as the ground truth. A quantitative comparison is carried out against methods which are considered as the representative state of the art for the regional image segmentation.

- Otsu multiregional hard thresholding (OTSU-N) proposed in Otsu (1979).

- $\quad$ Fuzzy C-means (FCM): performs segmentation using a clustering method proposed in Bezdek (984).

- The iterative thresholding segmentation (ITS) proposed in Wu et al. (2000).

The following scalar measures are considered for the retinal image quantitative comparison:

- Rand index $(R I)$ : it measures a similarity between two data clusters. $R I$ compares assignments between pairs of elements in two clusters on a base of a calculation of a fraction of the correctly classified elements against all the elements. $R I$ definition for $C_{1}$ and $C_{2}$ clusters is following: 


$$
R I\left(C_{1}, C_{2}\right)=\frac{2\left(n_{11}+n_{00}\right)}{N(N-1)}
$$

where $N$ denotes a total number of the points, $n_{11}$ denotes a number of the pairs that they are in the same cluster in $C_{1}$ and $C_{2}$ and $n_{00}$ is a number of the pairs belonging to different clusters. The $R I$ gives results in a range [0;1]. 0 indicates that the data clusters do not agree on any pair of the points, contrarily 1 indicates that the data clusters are completely same (Rand, 1971).

- Variation of information $(V I)$ : it measures a distance between two segmentations in a sense of the average conditional entropy described by:

$$
V I\left(C_{1}, C_{2}\right)=H\left(C_{1}\right)+H\left(C_{2}\right)-2 I\left(C_{1}, C_{2}\right)
$$

where $H\left(C_{i}\right)$ represents an entropy associated with the cluster, and $I$ represents a mutual information between $\mathrm{C}_{1}$ and $\mathrm{C}_{2}$ clusters (Meila, 2005).

- Receiver operating characteristic (ROC) curve: the ROC represents a classification model mapping instances between the data classes. In a quantitative comparison, we interpret the ROC curve by the area under curve (AUC) parameter giving the results in a range [0;1]. 1 indicates the best correlation between the segmentation results (Hastie et al., 2009).

- Mean squared error (MSE): it is an estimator measuring the average of the error squares between two segmentation results. The $M S E$ represents a risk function which corresponds with the expected value of the squared or quadratic error loss (Al-Najjar and Soong, 2012).

The $R I$ and $A U C$ measures greater is better, contrarily for $V I$ and $M S E$ smaller is better. The best result for each measurement is highlighted. The average for each tested parameter $(R I, V I, A U C)$ is considered in the following tables.

Table 4 Quantitative testing and comparison of the retinal image segmentation (retinal lesions)

\begin{tabular}{lcccc}
\hline & OTSU-N & $F C M$ & ITS & Active contours \\
\hline RI & 0.712 & 0.729 & 0.699 & 0.731 \\
VI & 2.991 & 2.871 & 3.412 & 2.873 \\
AUC & 0.881 & 0.921 & 0.798 & 0.952 \\
MSE & 31.118 & 29.365 & 32.598 & 23.548 \\
\hline
\end{tabular}

Note: For each configuration, the best result is italicised.

Table 5 Quantitative testing and comparison of the retinal image segmentation (OD)

\begin{tabular}{lcccc}
\hline & OTSU-N & $F C M$ & ITS & Active contours \\
\hline RI & 0.689 & 0.692 & 0.569 & 0.712 \\
VI & 2.871 & 2.870 & 3.551 & 2.871 \\
AUC & 0.874 & 0.897 & 0.655 & 0.912 \\
MSE & 33.255 & 31.215 & 33.655 & 25.211 \\
\hline
\end{tabular}

Note: For each configuration, the best result is italicised. 


\section{Conclusions}

Regarding a fact that a number of the premature infants recently have had a grown tendency, an early diagnostic of the RoP is an important step to prevent the child blindness. For a correct diagnostic it is necessary to physician be aware about a state of the immature retina. Therefore, it is necessary to achieve the best observations of the pathological spots on the retinal images in a comparison with the native retinal images. The retinal lesions extraction brings an objectification of the subjective diagnosis in a form of the feedback of a ration between the respective retinal lesions to the OD.

A model of the retinal lesions and the OD is based on a complex method comprising several steps. An important part is the retinal image preprocessing ensuring better image features of the retinal lesions and the OD. This part of the algorithm enhances the retinal object boundaries and contrast among the retinal objects and the image background. It is supposed that an effect of the segmentation is strongly depended on a quality of the input retinal records. Segmentation is carried out in a form of time evolving curve iteratively approximating an area of the retinal lesion and the OD. In a final step of the model, the binary classification is carried out. The snake model generates the energy map classifying the spatial image area into two parts depending on the energy convention. Inside of the snake, the energy is negative, contrarily outside, the energy is positive. By this selective approach we can classify the pixels belonging to the object being segmented.

From the clinical point of a view, the most important result of the segmentation is the geometrical features extraction of the retinal objects. For this task, we calculated an area and radius of the OD and the retinal lesions. Clinical experts suppose that the OD geometrical parameters should be stable over the time. It means that the OD can be considered from the first retinal image record. On the other hand the retinal lesions have tendency to spread over the time. Clinicians often track a time evolution of the retinal lesions subjectively in a comparison with the OD radius, or other geometrical features. The proposed model of the retinal structures allows for a modelling of the retinal lesions including the radius and the area extraction. This application brings objective feedback to clinical practice.

Lastly, we have compared an application of the active contour model on the RetCam 3 image records with other segmentation methods (OTSU-N, FCM and ITS) well representing the state of the art of the regional segmentation. A quantitative comparison has been carried out by the RI, VI, AUC and MSE parameters. Except for the VI parameter, the active contour method achieves the best results in a comparison with other methods. It can also be noted that the FCM method appears to be more effective than $O T S U-N$. This fact is probably caused by the hard thresholding utilised by the OTSU-N. The analysed retinal structures are often badly observable and detectable as well. It is especially caused by a lower resolution and contrasts therefore, the hard thresholding methods produce less accurate results. An important issue of the retinal image processing is also the retinal lesion brightness spectrum overlap with the retinal blood vessels. The multiregional segmentation methods often classify those retinal objects to a same class thus, the segmentation result is overestimated it is a cause of a less accuracy of the multiregional segmentation. Judging by the stated facts, the active contour method appears as suitable instrument for the retinal structures modelling. 


\section{Acknowledgements}

The work and the contributions were supported by the project SV4506631/2101 'Biomedicínské inženýrské systémy XII'. This study was supported by the research project The Czech Science Foundation (GACR) No. 17-03037S, Investment evaluation of medical device development.

\section{References}

Al-Najjar, Y.A.Y. and Soong, D.C. (2012) 'Comparison of image quality assessment: PSNR, HVS, SSIM, UIQI', International Journal of Scientific and Engineering Research, Vol. 3, No. 8, pp.1-5.

Awan, A.W., Awan, Z.W. and Akram, M.U. (2015) 'A robust algorithm for segmentation of blood vessels in the presence of lesions in retinal fundus images', IST 2015 - 2015 IEEE International Conference on Imaging Systems and Techniques, Proceedings, Art. No. 7294519.

Bezdek, J. (1984) 'FCM: the fuzzy c-means clustering algorithm', Comput. Geosci., Vol. 10, Nos. 2-3, pp.191-203.

Bibiloni, P., González-Hidalgo, M. and Massanet, S. (2016) 'A survey on curvilinear object segmentation in multiple applications', Pattern Recognition, Vol. 60, pp.949-970.

Christodoulidis, A., Hurtut, T., Tahar, H.B. and Cheriet, F. (2016) 'A multi-scale tensor voting approach for small retinal vessel segmentation in high resolution Fundus images', Computerized Medical Imaging and Graphics, Vol. 52, pp.28-43.

Dai, B., Wu, X. and $\mathrm{Bu}, \mathrm{W}$. (2017) 'Optic disc segmentation based on variational model with multiple energies', Pattern Recognition, Vol. 64, pp.226-235.

Fan, W-S., Chang, C-H., Horng, C-T., Yao, H-Y., Sun, H-Y., Huang, S-F., Wang, H-C. (2017) 'Human eye cataract microstructure modelling and its effect on simulated retinal imaging', Optics Communications, Vol. 385, pp.59-65.

Haq, Z., Andreoli, M.T. and Mieler, W.F. (2017) 'Dark retinal lesion in a young asymptomatic man', JAMA Ophthalmology, Vol. 135, No. 2, pp.155-156.

Hastie, T., Tibshirani, R. and Friedman, J.H. (2009) The Elements of Statistical Learning: Data Mining, Inference, and Prediction, 2nd ed., Springer Series in Statistics, Stanford, California.

Imani, E., Javidi, M. and Pourreza, H-R. (2015) 'Improvement of retinal blood vessel detection using morphological component analysis', Computer Methods and Programs in Biomedicine, Vol. 118, No. 3, pp.263-279.

Kirsali, P., Sambasivarao, K. and Badoni, P. (2014) 'An automatic system for the detection of optic disc and pathologies in retinal images', 2014 IEEE International Symposium on Signal Processing and Information Technology, ISSPIT 2014, Art. No. 7300593.

Kubicek, J., Bryjova, I. and Penhaker, M. (2016a) 'Macular lesions extraction using active appearance method', Lecture Notes of the Institute for Computer Sciences, Social-Informatics and Telecommunications Engineering, LNICST, Vol. 165, pp.438-447.

Kubicek, J., Timkovic, J., Augustynek, M., Penhaker, M. and Pokrývková, M. (2016b) 'Optical nerve disc segmentation using circual integro differential operator', Lecture Notes in Electrical Engineering, Vol. 362, pp.387-396.

Kubicek, J., Timkovic, J., Slonka, J., Penhaker, M., Augustynek, M. and Bryjova, I. (2016c) 'Optical nerve segmentation using the active shape method', Lekar a Technika, Vol. 46, No. 1, pp.13-20. 
Laaksonen, L., Hannuksela, A., Claridge, E., Fält, P., Hauta-Kasari, M., Uusitalo, H. and Lensu, L. (2016) 'Evaluation of feature sensitivity to training data inaccuracy in detection of retinal lesions', 2016 6th International Conference on Image Processing Theory, Tools and Applications, IPTA 2016, Art. No. 7820975.

Meila, M. (2005) 'Comparing clusterings: an axiomatic view', in Proc. of the 22nd International Conference on Machine Learning, New York, NY, USA, pp.577-584.

Morales, S., Engan, K., Naranjo, V. and Colomer, A. (2015) 'Retinal disease screening through local binary patterns', IEEE Journal of Biomedical and Health Informatics, Vol. 21, No. 1, pp.184-192.

Munk, M.R., Beck, M., Kolb, S., Larsen, M., Hamann, S., Valmaggia, C. and Zinkernagel, M.S. (2017) 'Quantification of retinal layer thickness changes in acute macular neuroretinopathy', British Journal of Ophthalmology, Vol. 101, No. 2, pp.160-165.

Nugroho, H.A., Oktoeberza, K.Z.W., Ardiyanto, I., Buana, R.L.B. and Sasongko, M.B. (2016) 'Automated segmentation of hard exudates based on matched filtering', Proceeding - 2016 International Seminar on Sensors, Instrumentation, Measurement and Metrology, ISSIMM 2016, Art. No. 7803728, pp.84-87.

Oshi, V., Agurto, C., Barriga, S., Nemeth, S., Soliz, P., Maccormick, I.J., Lewallen, S., Taylor, T.E. and Harding, S.P. (2017) 'Automated detection of malarial retinopathy in digital fundus images for improved diagnosis in Malawian children with clinically defined cerebral malaria', Scientific Reports, Vol. 7, No. 42703.

Otsu, N. (1979) 'A threshold selection method from gray-scale histogram', IEEE Trans. Syst., Man and Cyb., Vol. 9, No. 1, pp.62-66.

Patel, Y.R., Kirkman, M.S., Considine, R.V., Hannon, T.S. and Mather, K.J. (2017) 'Retinopathy predicts progression of fasting plasma glucose: an early diabetes intervention program (EDIP) analysis', Journal of Diabetes and its Complications, Vol. 31, No. 3, pp.605-610.

Rand, W.M. (1971) 'Objective criteria for the evaluation of clustering methods', J. Am. Stat. Assoc., Vol. 66, No. 336, pp.846-850.

Santhi, D., Manimegalai, D., Parvathi, S. and Karkuzhali, S. (2016) 'Segmentation and classification of bright lesions to diagnose diabetic retinopathy in retinal images', Biomedizinische Technik, Vol. 61, No. 4, pp.443-453.

Sharath Kumar, P.N., Deepak, R.U., Sasi, P.M., Rajesh Kumar, R., Sathar, A. and Sahasranamam, V. (2016) 'Automated severity scoring for diabetic retinopathy using Fundus photography', 2016 IEEE Annual India Conference, INDICON 2016, Art. No. 7838960.

Wu, H., Barba, J. and Gil, J. (2000) 'Iterative thresholding for segmentation of cells from noisy images', J. Microsc., Vol. 197, No. 3, pp.296-304.

Xiao, Z., Zhang, X., Zhang, F., Geng, L., Wu, J., Su, L. and Chen, L. (2017) 'Diabetic retinopathy retinal image enhancement based on gamma correction', Journal of Medical Imaging and Health Informatics, Vol. 7, No. 1, pp.149-154.

Xu, X., Liu, X., Wang, X., Clark, M.E., McGwin Jr., G., Owsley, C., Curcio, C.A. and Zhang, Y. (2017) 'Retinal pigment epithelium degeneration associated with subretinal drusenoid deposits in age-related macular degeneration', American Journal of Ophthalmology, Vol. 175, pp.87-98. 